\title{
Workplace-based rehabilitation of upper limb conditions: A Systematic Review
}

By: Munira Begum Hoosain

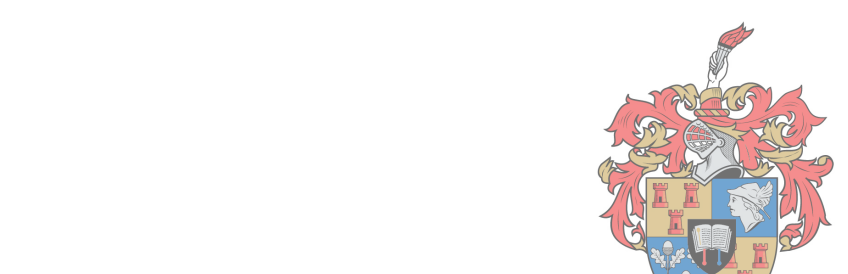

Thesis for Structured Masters by Publication presented in partial fulfilment of the requirements for the degree of

Master of Occupational Therapy

in the Faculty of Health Sciences at Stellenbosch University

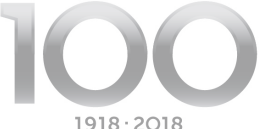

Supervisor: Susan de Klerk

Co-supervisor: Marlette Burger

March 2018 


\section{Declaration}

By submitting this thesis electronically, I declare that the entirety of the work contained therein is my own, original work, that I am the sole author thereof (save to the extent explicitly otherwise stated), that reproduction and publication thereof by Stellenbosch University will not infringe any third party rights and that I have not previously in its entirety or in part submitted it for obtaining any qualification.

Copyright @ 2018 Stellenbosch University All rights reserved 


\section{Abstract}

Background: Upper limb conditions are a common and growing cause of work related ill health and disability. International and South African legislation support work rehabilitation and specifically workplace-based rehabilitation, but the availability of workplace-based rehabilitation services appears to be limited in South Africa, with more focus on once-off work evaluations. Evidence is needed on the effectiveness of workplace-based rehabilitation services, in order to inform future service delivery.

Objective: The objective of this systematic review was to identify, collate and analyse the current available evidence on the effectiveness of workplace-based rehabilitative interventions in workers with upper limb conditions on work performance, pain, absenteeism, productivity and other outcomes such as ergonomic risk and mental health.

Methods: This systematic review was designed in accordance with PRISMA guidelines and registered with PROSPERO as number: PROSPERO CRD42017059708. We searched Medline (PubMed), Cochrane Library, Scopus, Web of Science, EBSCOhost (Academic Search Premier, Africa-Wide, CINAHL), OTSeeker and PEDro with search terms in four broad areas: upper limb, intervention, workplace and clinical trial (no date limits). Studies including neck pain only or musculoskeletal pain in other areas were not included. Risk of bias in included studies was assessed using a question and rating system developed by the Institute for Work and Health (IWH). As meta-analysis was not possible, study results were analysed through a narrative synthesis.

Results: The initial literature search located 1071 articles, of which 80 were full text reviewed. Seventeen studies were included, across 28 articles, reporting on various outcomes. Nine studies were of high methodological quality, seven of medium quality, and one of low quality. Studies were sorted into intervention categories: Ergonomic controls $(n=3)$, ergonomic training and workstation adjustments $(n=4)$, exercise and resistance training $(n=6)$, clinic-based versus 
workplace-based work hardening $(n=1)$, nurse case manager training $(n=1)$, physiotherapy versus Feldenkrais $(n=1)$, and ambulant myofeedback training $(n=1)$. The largest body of evidence supported workplace exercise programs, with positive effects for ergonomic training and workstation adjustments, and mixed effects for ergonomic controls. Ambulant myofeedback training had no effect. The remaining three categories had positive effects in the single study on each intervention.

Conclusion and Recommendations: There is substantial evidence supporting workplace exercise programs. Further research needs to be conducted on the remaining intervention categories. Researchers are encouraged to collaborate with clinicians to enable more high quality research in "real-life" rehabilitation contexts, including individualised work rehabilitation. Clinicians should build partnerships with the Department of Labour and stakeholders at workplaces, in order to develop rehabilitation resources in work environments. 


\section{Opsomming}

Agtergrond: Boonste ledemaat toestande is ' $\mathrm{n}$ algemene en toenemende oorsaak van werkverwante siektes en gestremdheid. Internasionale en Suid-Afrikaanse wetgewing ondersteun werkrehabilitasie, veral rehabilitasie wat by die werkplek plaasvind. In Suid-Afrika is rehabilitasie by die werkplek nog beperk, met meer fokus op eenmalige werk evaluerings. Inligting oor die doeltreffendheid van werkplek gebaseerde rehabilitasie dienste is nodig ten leiding aangaande toekomstige dienslewering.

Doelwit: Die doelwit van hierdie sistematiese oorsig was om die huidige beskikbare navorsing oor die effektiwiteit van werkplek gebaseerde rehabilitasie dienste in werkers met boonste ledemaatstoestande op werkverrigting, pyn, afwesigheid, produktiwiteit en ander uitkomste soos ergonomiese risiko en geestesgesondheid, te identifiseer, saam te voeg en te analiseer.

Metode: Hierdie sistematiese oorsig is volgens PRISMA riglyne ontwerp en by PROSPERO geregistreer: PROSPERO CRD42017059708. Ons het gesoek in Medline (PubMed), Cochrane Biblioteek, Scopus, Web of Science, EBSCOhost (Academic Search Premier, Africa-Wide, CINAHL), OTSeeker en PEDro met sleutelwoorde in die volgende vier areas: boonste ledemaat, intervensie, werkplek en kliniese proef (met geen datum limiete). Studies oor slegs nekpyn of muskuloskeletale pyn in ander gebiede, is nie ingesluit nie. Risiko van vooroordeel in ingeslote studies is beoordeel deur ' $n$ vraag- en graderingstelsel wat deur die Instituut vir Werk en Gesondheid (Institute for Work and Health (IWH)) ontwikkel is. Aangesien 'n meta-analise nie moontlik was nie, is studie-uitslae geanaliseer deur middel van 'n narratiewe sintese.

Resultate: Die aanvanklike literatuursoektog het 1071 artikels opgetref, waarvan 80 volledige artikels ge-evalueer is. Sewentien studies is ingesluit, vanuit 28 artikels wat oor verskeie uitkomste verslag lewer. Nege studies was van hoë metodologiese gehalte, sewe van medium gehalte en een van lae gehalte. Studies is gesorteer in intervensie kategorieë: Ergonomiese kontrole $(n=3)$, ergonomiese opleiding en werkstasie aanpassings $(n=4)$, oefening en 
weerstandsopleiding $(n=6)$, kliniek gebaseerde teenoor werkplek gebaseerde werkverharding $(n=1)$, verpleegkundige bestuurder opleiding $(n=1)$, fisioterapie teenoor Feldenkrais $(n=1)$, en ambulante myoterugvoer opleiding $(n=1)$. Werkplek oefenprogramme is deur die meeste navorsing ondersteun, met positiewe gevolge vir ergonomiese opleiding en werkstasie aanpassings, en gemengde effekte vir ergonomiese kontrole. Ambulante myoterugvoer opleiding het geen effek getoon nie. Die oorblywende drie kategorieë het positiewe effekte getoon in die enkele studie oor elke intervensie.

Gevolgtrekking en Aanbevelings: Werkplek-oefenprogramme word goed deur navorsing ondersteun. Verdere navorsing moet gedoen word oor die oorblywende intervensie kategorieë. Navorsers word aangemoedig om met terapeute saam te werk, ten einde meer hoë kwaliteit navorsing in rehabilitasie omgewings te doen, insluitende geïndividualiseerde werkrehabilitasie. Terapeute moet met die Departement van Arbeid en belanghebbendes by werksplekke vennootskappe bou, om rehabilitasie hulpbronne in werksplekke te ontwikkel. 


\section{Acknowledgements}

My most grateful thanks firstly to the Almighty Allah for guiding me to this Masters program, which has been a blessing in my life for the past five years. I am confident that the skills and perspective gained will be beneficial to me in various contexts. It has been a rewarding and exciting journey that I have never for a moment regretted.

Heartfelt thanks to my supervisors, Susan and Marlette, for their warmth, enthusiasm, technical and emotional support. They were always available to me when needed, motivating and encouraging me till the end. I have learnt so much from both of you - knowledge of systematic review process and good scientific practice, skills to conduct and write up this study and a positive attitude towards research and publication. I wish you everything of the best in your own research and any future endeavours. May you both be blessed.

Thanks to my wonderfully supportive husband, for many hours of listening to me ramble on about research (much of which must have been incredibly boring to him), for showing an interest, for encouraging me, for supporting me emotionally and practically, and for his pride in my every little accomplishment towards the end of this project.

Thanks to my dear friend Jamiela, my partner in juggling studies with managing a household and raising children. This project was made so much easier and more pleasurable by sharing the process with you.

Thanks to my children, Aaliyah, Yunus and Kauthar, for sharing your mother with her research and for your excitement about my studies. I look forward to guiding and supporting each of you through your own higher education.

Thanks to the occupational therapy department at Stellenbosch University, particularly Zelda, Lana and Elri. You have all been instrumental in this journey. Thanks to Ingrid for assistance in designing the literature search strategy, and for your patience in teaching me and replying to my many emails. 


\section{Table of Contents}

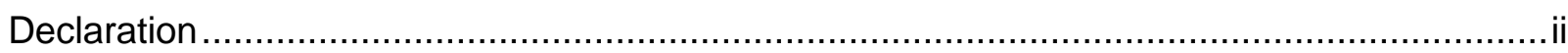

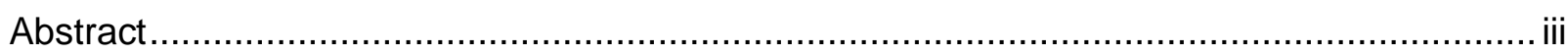

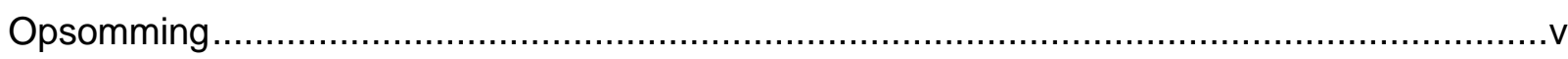

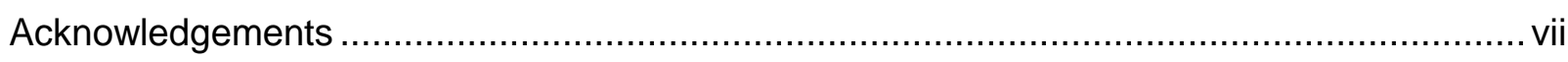

Table of Contents .......................................................................................... vii

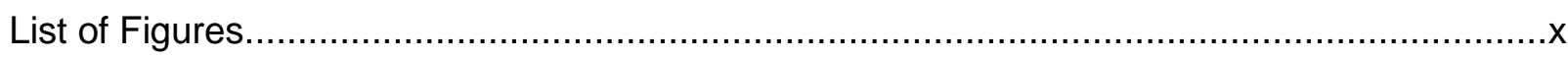

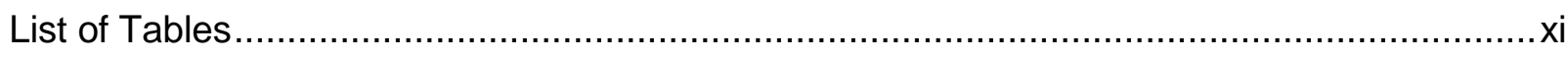

List of Abbreviations .................................................................................................

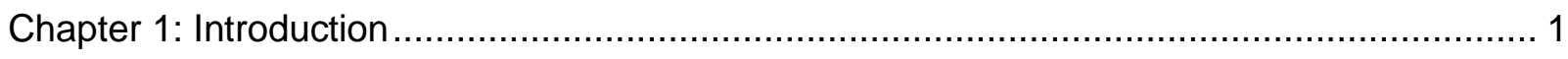

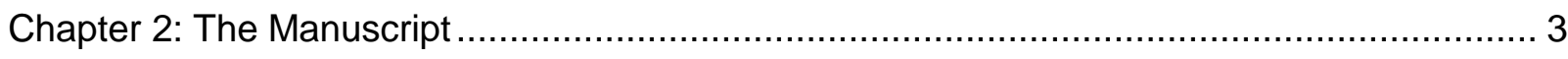

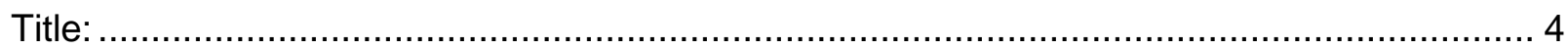

Authors:

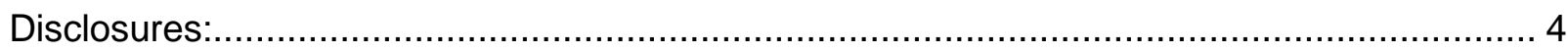

Abstract:

Keywords

Background:

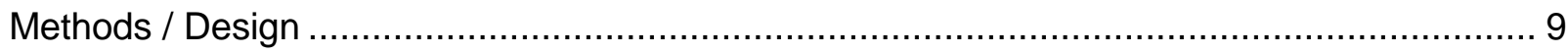

Study design

Eligibility criteria

Type of studies

Type of participants

Type of interventions

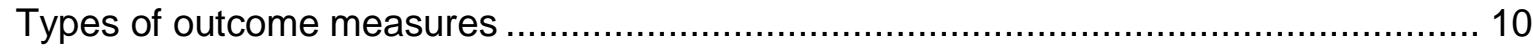

Information sources and search strategy ……….................................................. 10

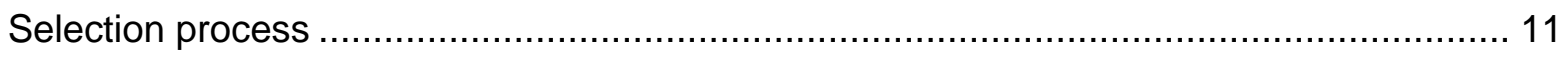

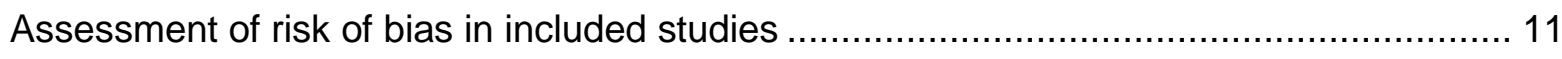

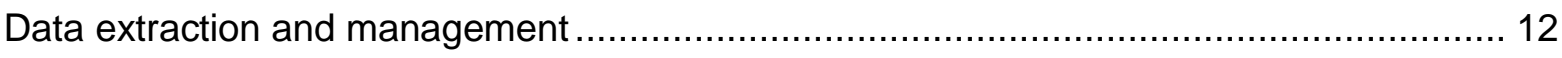

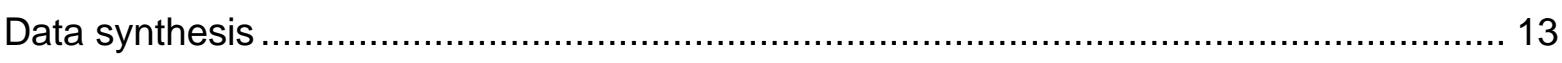

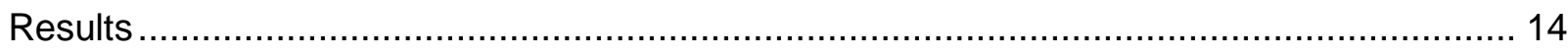

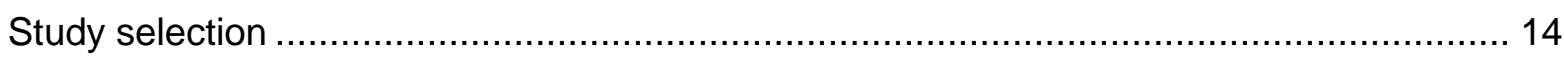

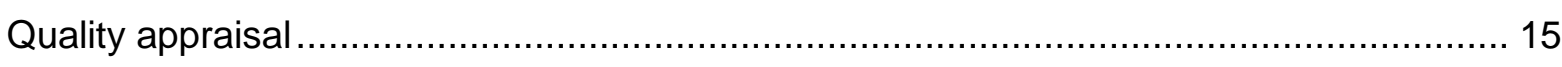




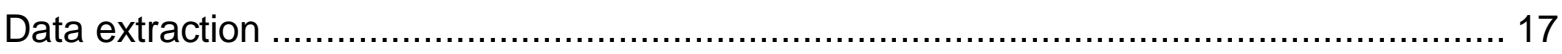

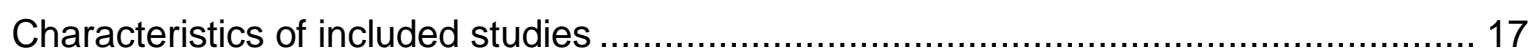

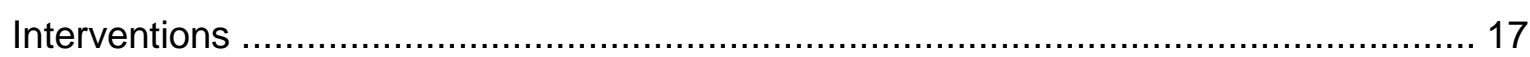

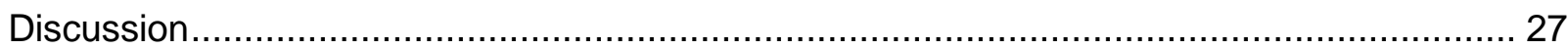

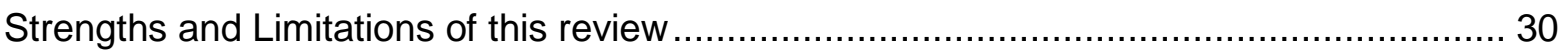

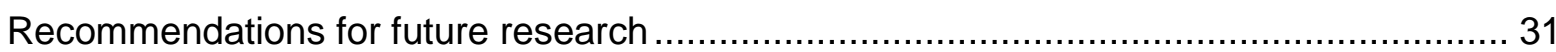

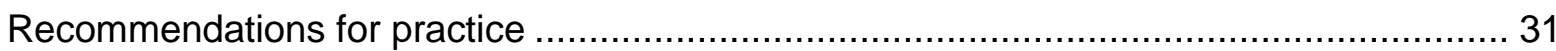

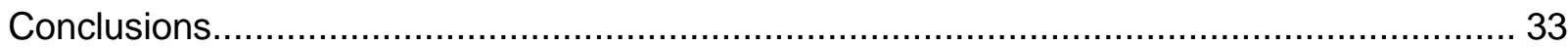

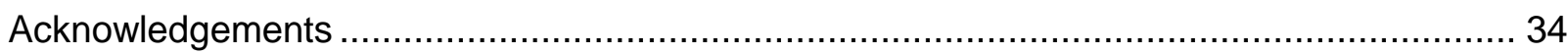

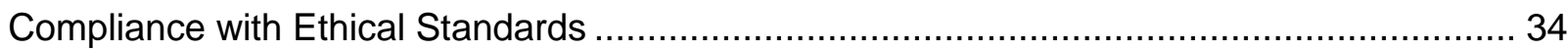

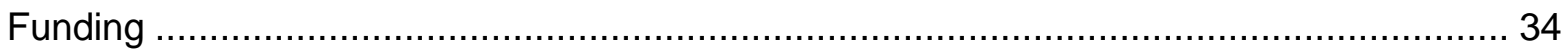

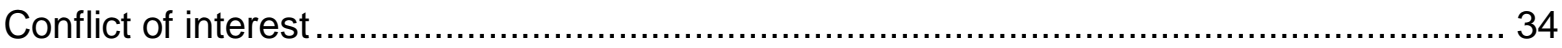

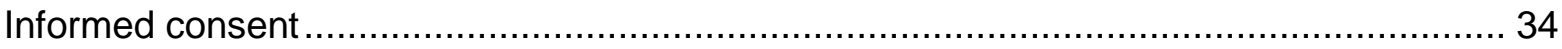

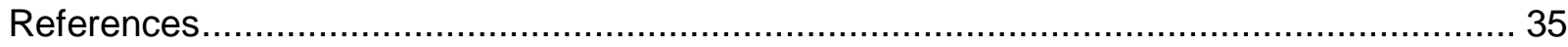

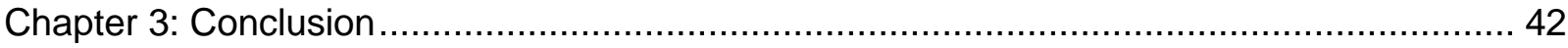

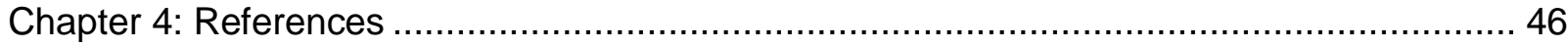

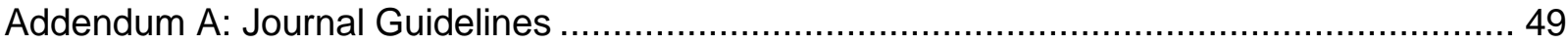

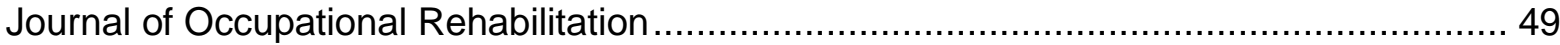

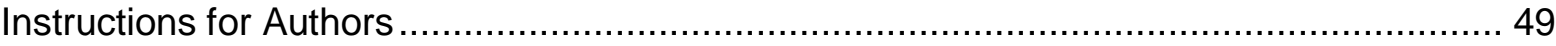

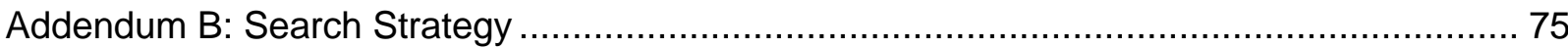

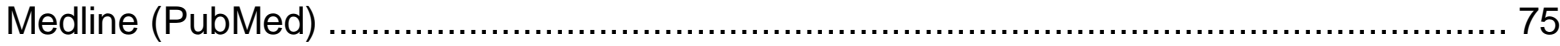

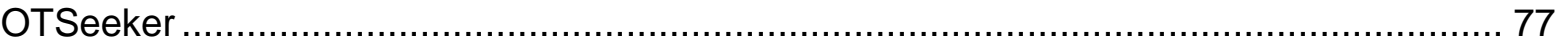

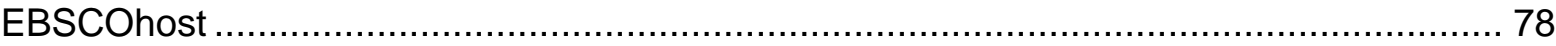

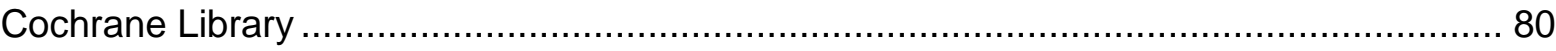

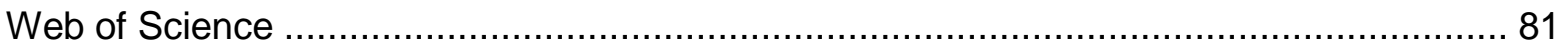

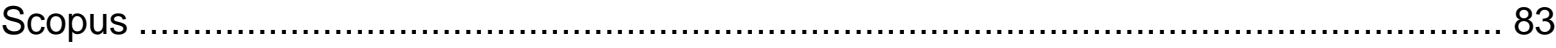

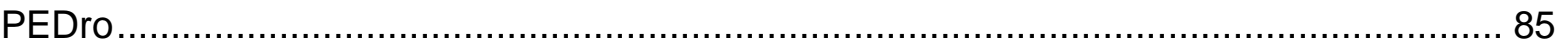

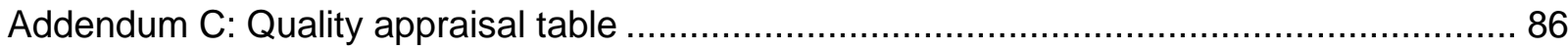




\section{List of Figures}

Figure 1: PRISMA flow diagram 


\section{List of Tables}

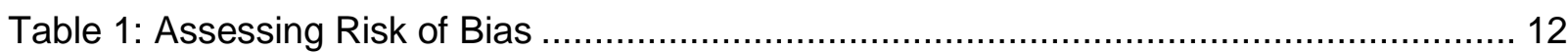

Table 2: Quality Appraisal and Characteristics of Studies............................................... 16

Table 3: Description of Interventions, Main Outcomes and Findings ................................. 19 


\section{List of Abbreviations}

\begin{tabular}{|c|c|c|}
\hline RSI & - & Repetitive strain injury \\
\hline EBP & - & Evidence based practice \\
\hline USA & - & United States of America \\
\hline WRUED & - & Work-related upper extremity disorders \\
\hline $\mathrm{RCT}$ & - & Randomised controlled trial \\
\hline EMG & - & Electromyograph \\
\hline DASH & - & Disabilities of the arm, shoulder and hand questionnaire \\
\hline SF-36 & - & Short form (36) health survey \\
\hline WHODAS & - & World Health Organisation Disability Assessment Schedule \\
\hline $\mathrm{MeSH}$ & - & Medical subject headings \\
\hline IWH & - & Institute for Work and Health \\
\hline I & - & Intervention \\
\hline C & - & Control \\
\hline$P$ & - & Protocol \\
\hline$B G$ & - & Between group \\
\hline MS & - & Musculoskeletal \\
\hline RULA & - & Rapid Upper Limb Assessment \\
\hline HAL & - & Hand activity level \\
\hline UE & - & Upper extremity \\
\hline
\end{tabular}




\begin{tabular}{|c|c|c|}
\hline SSS & - & Symptom severity scale \\
\hline FSS & - & Functional status scale \\
\hline SS & - & Statistically significant \\
\hline APB & - & Abductor pollicis brevis test \\
\hline NMQ & - & Nordic musculoskeletal questionnaire \\
\hline VAS & - & Visual analogue scale \\
\hline UEFS & - & Upper extremity functional scale \\
\hline UED & - & Upper extremity disorders \\
\hline PRT & - & Progressive resistance training \\
\hline RPE & - & Rating of perceived exertion \\
\hline WAI & - & Work ability index \\
\hline SPADI & - & Shoulder pain and disability index \\
\hline QPS NORDIC & - & $\begin{array}{l}\text { General Nordic questionnaire for psychological and social } \\
\text { factors at work }\end{array}$ \\
\hline FCE & - & Functional capacity evaluation \\
\hline ROM & - & Range of motion \\
\hline $\mathrm{OTOH}$ & - & Occupational Therapy in Occupational Health \\
\hline WPG & - & Work Practice Group \\
\hline WFOT & - & World Federation of Occupational Therapy \\
\hline ILO & - & International Labour Organisation \\
\hline WHO & - & World Health Organisation \\
\hline
\end{tabular}




\section{Chapter 1: Introduction}

Upper limb conditions are amongst the most common causes of ill-health and disability in the workplace [1,2]. A cross-sectional field survey of South African employees ( $n=15663)$ in 2012 found that $47 \%$ of employees experienced repetitive strain injury (RSI)-related symptoms in their neck, shoulder and upper back [3]. While all provinces and races were represented, the sample consisted of educated participants, ranging from Grade 8 to doctoral degree. This is unlikely to be fully representative of the South African workforce. The incidence of upper limb pain amongst South African workers with lower educational levels could possibly be higher, particularly in those involved with manual labour or highly repetitive unskilled or semi-skilled work. The impact of upper limb dysfunction on the South African workforce includes direct costs of compensation for work-related injuries by the Compensation Fund, loss of productivity and work quality, absenteeism, worker retraining and replacement [4].

International and South African legislation are clearly supportive of work rehabilitation, specifically workplace-based rehabilitation (interventions offered directly at the workplace, rather than at hospitals or rehabilitation centres). Articles 26 and 27 of the United Nations Convention on the Rights of Persons with Disabilities recognises the rights of persons with disabilities to work on an equal basis with others [5]. To this end, the policy promotes work rehabilitation and return-to-work programmes for persons with disabilities. The International Labour Office Vocational Rehabilitation and Employment (Disabled Persons) Recommendation states that work rehabilitation should be started as early as possible [6]. This policy also advocates for the contribution of employers' and workers' organisations to the development of work rehabilitation services. The Integrated National Disability Strategy stresses the importance of work rehabilitation in enabling people with disabilities to retain employment and becoming fully participating members of society [7]. The South African Compensation Fund's 2014-2019 Strategic Plan identifies the development of a Rehabilitation, Reintegration and Return-to-Work Policy Framework as a key priority [8]. 
Despite the need and legislative support for work rehabilitation services, a recent descriptive cross-sectional study $(n=109)$ [9] found that $72 \%$ of South African occupational therapists in the field of work practice offered no treatment or rehabilitation services, instead focussing on onceoff evaluations. Furthermore, only $1 \%$ of practitioners were based at industrial settings, with the overwhelming majority practicing at hospitals, work assessment units or work rehabilitation units. However, it was found that a small percentage of practitioners ( $35 \%$ or less) occasionally offered some services at workplaces. These included supported employment, job coaching and support, wellness/fitness programs and symptom/discomfort screenings. When offered, work rehabilitation services were more commonly situated at clinics or therapists' practices - these included work conditioning and job modification, joint protection and energy conservation programs, and re-integration programs [9].

Evidence based practice (EBP) challenges health professionals to practice ethically and to consider clinical efficacy [10], using research results in everyday clinical practice. While challenges persist in practical implementation of EBP amongst health professionals in high income [11], as well as middle and lower income countries [12], the EBP process is supported by access to pre-appraised evidence, which is facilitated by high quality systematic reviews [10].

An up-to-date systematic review on effective rehabilitative workplace interventions for upper limb conditions would assist in guiding practitioners in the selection and structuring of suitable workplace-based programs. Such a review would take account of what type of interventions are available internationally, which practitioners they are offered by and what outcomes are successfully improved by these interventions. This would guide the development of evidencebased workplace interventions in South Africa, in order to address the growing problem of upper limb conditions in the workplace, and bridge the current gap between legislation and practice.

The aim of this study was therefore to determine the effectiveness of workplace-based rehabilitative interventions in workers with upper limb conditions on work performance, pain, absenteeism, productivity and other outcomes, including ergonomic risk and mental health. 


\section{Chapter 2: The Manuscript}

This manuscript is to be submitted to the Journal of Occupational Rehabilitation.

The Journal Guidelines for Authors are included as Addendum A.

Please note that referencing in the manuscript is independent of the rest of the research report. 


\section{Title:}

Workplace-based rehabilitation of upper limb conditions: A Systematic Review

\section{Authors:}

Munira Hoosain, Division Occupational Therapy, Faculty of Medicine and Health Science, Stellenbosch University. Email: munirahoosain@gmail.com

Susan de Klerk, Division Occupational Therapy, Faculty of Medicine and Health Science, Stellenbosch University. Email: sdk@sun.ac.za

Marlette Burger, Division Physiotherapy, Faculty of Medicine and Health Science, Stellenbosch University. Email: mbu@sun.ac.za

Corresponding author: Munira Hoosain. Division Occupational Therapy, Faculty of Medicine and Health Science, Stellenbosch University. Email: munirahoosain@gmail.com. Tel: +27 835662970.

\section{Disclosures:}

The authors declare that they have no conflict of interest. No funding was obtained for this study. 


\section{Abstract:}

Purpose: The objective of this systematic review was to identify, collate and analyse the current available evidence on the effectiveness of workplace-based rehabilitative interventions in workers with upper limb conditions on work performance, pain, absenteeism, productivity and other outcomes.

Methods: We searched Medline (PubMed), Cochrane Library, Scopus, Web of Science, Academic Search Premier, Africa-Wide Information, CINAHL, OTSeeker and PEDro with search terms in four broad areas: upper limb, intervention, workplace and clinical trial (no date limits). Studies including neck pain only or musculoskeletal pain in other areas were not included.

Results: Initial search located 1071 articles, of which 80 were full text reviewed. Seventeen studies were included, on which 28 articles reporting on various outcomes were found. Nine studies were of high methodological quality, seven of medium quality, and one of low quality. Studies were sorted into intervention categories: Ergonomic controls $(n=3)$, ergonomic training and workstation adjustments $(n=4)$, exercise and resistance training $(n=6)$, clinic-based versus workplace-based work hardening $(n=1)$, nurse case manager training $(n=1)$, physiotherapy versus Feldenkrais $(n=1)$, and ambulant myofeedback training $(n=1)$. The largest body of evidence supported workplace exercise programs, with positive effects for ergonomic training and workstation adjustments, and mixed effects for ergonomic controls. Ambulant myofeedback training had no effect. The remaining three categories had positive effects in the single study on each intervention.

Conclusion: While there is substantial evidence for workplace exercise programs, other workplace-based interventions require further high quality research.

Systematic review registration: PROSPERO CRD42017059708

Keywords: Workplace, Rehabilitation, Upper extremity, Occupational health 


\section{Background:}

Upper limb conditions, whether work related or not, continue to pose significant challenges in the workplace. Repetitive strain injury $(\mathrm{RSI})$ is the most common cause of work-related ill health internationally [1]. In high income economies such as the United States of America (USA), Canada and West Europe, upper limb and lower back disorders are among the leading occupational injuries and diseases, and considered a growing problem [2]. Similarly, in middle and lower income economies, musculoskeletal disorders are among the most commonly reported work-related diseases [3].

Workplace-based rehabilitation services may be offered by a variety of healthcare providers, including occupational therapists, physiotherapists and ergonomists. Services may include workplace-based exercise programs [4,5], education of workers [6], modifications to work stations or work process [4], rest breaks [4], and training of supervisors or case managers [4].

Rehabilitation services based at the workplace may have specific advantages over traditional rehabilitation, based at hospitals and rehabilitation centres. Some of these advantages include reduced time off work, earlier return to work, improved quality of life and reduced cost of injuries [4]. Situating rehabilitation services onsite allows injured workers earlier access to rehabilitation, with the potential for better outcomes $[7,8]$. The role of workplace supervisors in work rehabilitation is well recognised. Early contact between healthcare workers and the workplace has been found to facilitate a reduction in the duration of work disability $[6,8]$. Healthcare workers based at the workplace may be better placed to train supervisors and negotiate work accommodations; factors which are associated with improved rehabilitation outcomes [8].

A systematic review investigating the effectiveness of workplace-based rehabilitation interventions in the treatment of work-related upper extremity disorders (WRUEDs) was conducted in $2004(n=8)$ [4]. Eight studies met the review's inclusion criteria. These included four randomised controlled trials (RCTs), three cohort studies and one case series. Interventions 
included individual physiotherapy at a local clinic, group exercise at the workplace, physiotherapy based at the workplace, worksite analysis, a training program for nursing case managers on workplace accommodations, ergonomic modifications, as well as rest and exercise breaks. The review concluded that although some positive findings supported the effectiveness of workplace-based interventions in rehabilitating WRUEDs, poor study design affected the reliability of these findings. The flaws in design of individual studies included small sample sizes, lack of standardised outcome measures and statistical analyses, poor reporting of interventions and results, and failure to include control groups. The researchers acknowledged the difficulty in performing workplace-based interventions, and considering this, felt that the risk of bias assessments utilised in the review (Sackett's levels of evidence [9] and the Evaluation Guidelines for Rating the Quality of an Intervention Study Form [10]) may have been overly rigorous. Recommendations included the development of a set of core outcome measures with tested psychometric properties, development of sound methodology for conducting workplace studies, and improved reporting of interventions and study designs [4]. A more recent systematic review was published in 2010 , with an update in 2016 , on the effectiveness of workplace-based interventions in the prevention of upper limb conditions [2,11]. The initial review included 36 studies of medium to high quality; of which 23 were RCTs, eight were non-randomised trials, and five were cross-over designs [11]. The 2016 update identified an additional 26 medium to high quality studies, of which nine were RCTs, 12 were cluster RCTs and five were non-randomised trials with a control group [2]. Data from the two reviews were combined and grouped into intervention categories. Meta-analysis was not conducted due to differences between comparison/control groups, varied outcome measures and insufficient data reported. Strong evidence was found to support workplace-based resistance training exercise programs; while moderate evidence of positive effect was found for forearm supports, vibration feedback on static mouse use and stretching exercise programs. There was moderate evidence of no effect for EMG biofeedback, job stress management training and office workstation adjustment. Insufficient or conflicting evidence was found for the remaining 23 intervention groups. The updated review found a large number of studies over a shorter period of time than 
the initial review, and a larger proportion of the more recent studies (from 2008 onward) were of higher quality. Limitations of the review include the inability to include a meta-analysis and the risk of publication bias, as grey literature was not included [2].

While legislation supports workplace-based rehabilitation, a systematic review of existing research on the topic would be beneficial in determining effectiveness of this type of intervention. An up to date systematic review of workplace-based rehabilitative interventions for upper limb conditions would be valuable in accounting for all subsequent literature in this field, particularly considering the value of the 2016 updated review on preventative interventions for upper limb conditions. This review would serve to guide evidence based practice amongst occupational therapists and other rehabilitation professionals working with upper limb conditions in the workplace.

The objective of this review was to determine the effectiveness of workplace-based rehabilitative interventions in workers with upper limb conditions on work performance, pain, absenteeism, productivity and other outcomes, including ergonomic risk and mental health. 


\section{Methods / Design:}

\section{Study design}

This review adheres to the Preferred Reporting Items for Systematic Review and Meta-analysis (PRISMA-P) [12,13]. The review protocol was registered with the International Prospective Register of Systematic Reviews (PROSPERO: CRD42017059708).

\section{Eligibility criteria}

\section{Type of studies}

All clinical intervention studies were considered, including randomised and non-randomised clinical trials published from inception of the databases until April 2017.

\section{Type of participants}

Adults aged 18 years or older who are actively employed, with any upper limb condition, including WRUEDs, traumatic injury, degenerative conditions and non-specific or undiagnosed upper limb pain.

\section{Type of interventions}

Rehabilitation programs that included any workplace-based interventions were included in the review. Interventions were all at least partly based at the workplace. Interventions based at occupational health clinics were included. Studies on off-site work rehabilitation interventions only (e.g. based at rehabilitation centres, hospitals, therapy clinics, work hardening programs) were excluded.

Examples of workplace-based interventions included job task adaptations, job rotation or alternate placement, work environment/work station or alternate ergonomic modifications, stretching/exercise programs, implementation of rest breaks at work, work hardening, 
negotiation with supervisors or managers, splint application at work, worker education and supervisor or manager education. Interventions with or without controls were included.

\section{Types of outcome measures}

\section{Primary outcomes}

The primary outcome of interest was work performance, as measured by productivity, absenteeism, pain or comfort at work, satisfaction or motivation at work.

\section{Secondary outcomes}

Outcomes related to upper limb function which are not necessarily related to work, including but not confined to the Disabilities of the Arm, Shoulder and Hand (DASH) questionnaire, grip strength, range of motion; health-related quality of life measures, e.g. SF-36, WHODAS 2.0; or standardised measures of participation in activities of daily living, e.g. Barthel Index.

\section{Information sources and search strategy}

With the help of an expert librarian we designed and conducted a search strategy through Stellenbosch University Library and Information Service to find eligible articles in a combination of generalist and specialist electronic databases from March to April 2017, including: Cochrane Central Register of Controlled Trials (CENTRAL) in the Cochrane Library; Medline (PubMed); Scopus; Web of Science; EBSCOhost (Academic Search Premier, Africa-Wide, CINAHL); OTSeeker and PEDro. No language exclusions or date limits were applied. Search strings were adapted by database, and included: exploded MeSH terms, free text, subheadings, synonyms and variant spellings, lay and medical terminology, truncation, Boolean operators, AND and OR. The complete list of terms and detailed search strategy are included as Addendum B. The reference lists of included studies were hand searched for further studies. Full text articles were obtained for all potentially eligible titles. The search results were uploaded into the online software package, Covidence (www.covidence.org), for removal of duplicates (April 2017). Covidence was used for both abstract (April to May 2017) and full-text screening (June 2017). 
The total number of results before and after removal of duplicates were documented in a PRISMA flow diagram (See Figure 1).

\section{Selection process}

Articles were initially screened for relevance by title and abstract. Thereafter, the full text of potentially suitable studies were retrieved, and inclusion criteria applied. Inclusion criteria comprised articles based on primary empirical research, i.e. not a review, letter to a journal editor, opinion piece or editorial; clinical trials; articles reporting work rehabilitation interventions that were workplace-based, aimed at adult workers with pre-existing upper limb symptoms. Exclusion criteria comprised samples of workers with neck symptoms only; no clear evidence of pre-existing upper limb pain in the sample; other musculoskeletal conditions included in the sample. The reference lists of included articles were screened for additional titles. Title and abstract screening was conducted by the principal investigator $(\mathrm{MH})$. Full text article screening was conducted independently by both $\mathrm{MH}$ and a second reviewer (SdK), in order to limit bias in the review (June to July 2017). Disagreements were resolved through discussion. Search dates were recorded. The process followed in screening and selection of studies was reported in a PRISMA flow diagram (Figure 1) [14,15].

\section{Assessment of risk of bias in included studies}

Included studies were assessed for risk of bias by the principal investigator (and first reviewer) $(\mathrm{MH})$ and second reviewer (SdK), independently. Results of the independent reviews were correlated, with discussion to reach consensus on any differences. A third reviewer (MB) was available to resolve conflicts in case consensus could not be reached, but this was not needed.

Risk of bias was assessed using the question and rating system developed by the Institute for Work and Health (IWH) and used in their systematic review on occupational health and safety interventions preventing upper limb symptoms [2,11] (Table 1). As in the above review, a 3- 
point rating system was used to qualify the questions, ranging from "somewhat important" (1), to "very important" (3) (See Table 1).

Table 1: Assessing Risk of Bias

\begin{tabular}{|c|c|}
\hline Question & Rating \\
\hline 1. Is the research question clearly stated? & 2 \\
\hline 2. Were comparison group(s) used? & 3 \\
\hline 3. Was an intervention allocation described adequately? & 3 \\
\hline 4. Was recruitment/participation rate reported? & 2 \\
\hline 5. Were pre-intervention characteristics described? & 2 \\
\hline 6. Was attrition less than $35 \%$ ? & 2 \\
\hline $\begin{array}{l}\text { 7. Did the author examine for important differences between the remaining and drop-out } \\
\text { participants after the intervention? }\end{array}$ & 2 \\
\hline 8. Was the intervention process adequately described to allow for replication? & 3 \\
\hline 9. Were the effects of the intervention on some exposure parameters documented? & 1 \\
\hline 10. Was the participation in the intervention documented? & 2 \\
\hline $\begin{array}{l}\text { 11. Were the upper extremity musculoskeletal symptoms, signs, disorders, injuries, claims and/or } \\
\text { lost time outcomes described at baseline and at follow-up? }\end{array}$ & 3 \\
\hline 12. Was the length of follow-up three months or greater? & 2 \\
\hline $\begin{array}{l}\text { 13. Was there adjustment for pre-intervention differences (minimum threshold of three important } \\
\text { covariates include age, gender and primary outcome at baseline)? }\end{array}$ & 3 \\
\hline 14. Were the statistical analyses optimized for the best results? & 3 \\
\hline $\begin{array}{l}\text { 15. Were all participants' outcomes analysed by the groups to which they were originally allocated } \\
\text { (intention-to-treat analysis)? }\end{array}$ & 2 \\
\hline 16. Was there a direct between-group comparison? & 3 \\
\hline
\end{tabular}

1=somewhat important, 2=moderately important, 3=very important

A maximum score of 41 is possible, using these 16 criteria. As in the above IWH review, studies were grouped into three categories based on their quality ranking score: High quality $(>85 \%)$, Medium quality (50-85\%) and low quality $(<50 \%)[2,11]$.

\section{Data extraction and management}

Information was extracted from each study (including low quality studies) by the principal investigator and entered into electronic data collection tables on Microsoft Excel. Data was extracted on items including study methods; demographic information of participants; interventions in terms of type, provider, duration, amount of treatment sessions, location and controls; outcomes; conflicts of interest and funding sources. Data entry was double checked 
for accuracy by the principal investigator on two separate dates. Spot accuracy checks were conducted by the second reviewer (SdK) and third reviewer (MB) on 50\% of included studies.

\section{Data synthesis}

Results were stratified by intervention type, and further by frequency/duration of intervention, intervention provider and outcome measure.

Due to the heterogeneity of the interventions, comparisons used, reporting of outcome measures and statistical analyses in the included studies, statistical pooling of data in the form of a meta-analysis was not appropriate for this review. Results were subsequently summarized and tabulated in the narrative form. 


\section{Results}

\section{Study selection}

A total of 1071 titles were found on the initial search, including 272 titles from Medline, 172 from OTSeeker, 97 from EBSCOhost, 50 from the Cochrane library, 50 from Web of Science, 420 from Scopus and 11 from PEDro (see Figure 1). Of these, 155 were duplicates, leaving 916 titles for screening. The initial screening of titles and abstracts excluded 808 irrelevant titles. Three of these titles were not available in English (one Norwegian, one Lithuanian and one Afrikaans). Google Translate was used to translate the titles and abstracts. A second title and abstract screening step was conducted jointly by the principal investigator and second reviewer, through discussion. Inclusion criteria were discussed and clarified, and an additional 28 titles excluded.

Full text review was then conducted independently by the principal investigator and second reviewer on 80 studies, resulting in the exclusion of 55 studies. The main reason for exclusion was that participants were not exclusively workers with pre-existing upper limb conditions or pain - studies included asymptomatic workers, or workers with musculoskeletal pain in other regions. One study was excluded as the article was available in Polish only, and could not be translated. Twenty-five articles proceeded to inclusion, and an additional three articles were identified through scanning of reference lists. Several of these 28 articles related to the same research and authors, reporting on different outcomes for the same intervention and control groups, and were thus grouped into 17 studies for inclusion in this review. Protocols of included studies that were published separately were included, to ensure that all available information was used for the quality appraisal. 


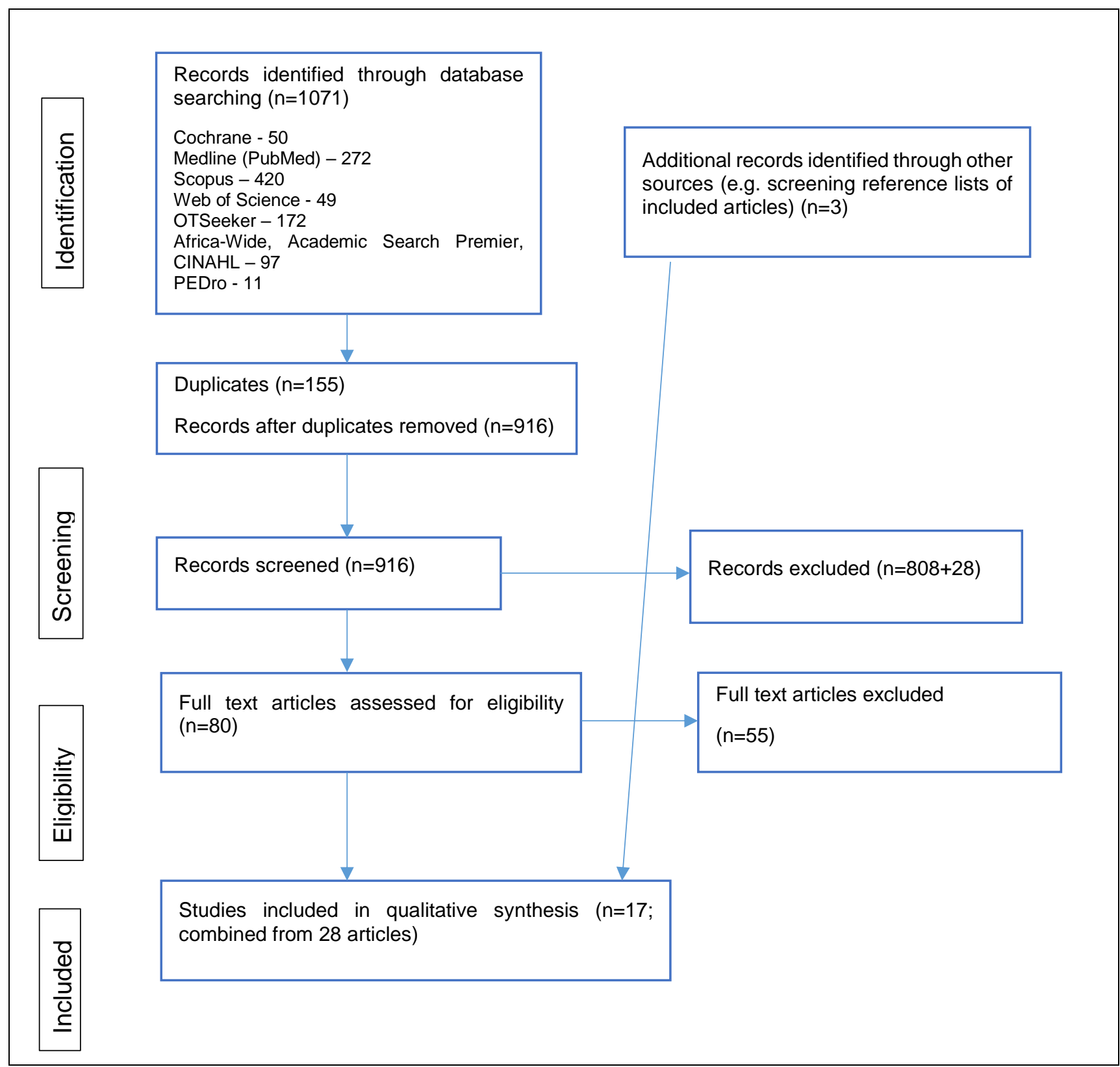

Fig. 1 PRISMA flow diagram

\section{Quality appraisal}

Table 2 depicts the list of included studies, their quality appraisal and characteristics. Nine studies were classified as high quality (meeting $>85 \%$ of criteria) [16-32], seven studies were classified as medium quality (meeting $50-85 \%$ of criteria) [32-42], and one study was classified as low quality $(<50 \%$ of criteria met) [43]. Medium quality studies did not perform intention-totreat analysis $(n=7)[32,36-38,40,42,43]$, did not document participation in the intervention adequately $(n=6)[33-37,39-42]$, had a follow-up length that was less than 3 months or unclear $(n=5)[32,36,39-42]$, and did not report on important differences between dropouts and 
remaining participants after the intervention $(n=4)[36-38,42]$. The one low quality study [43] had no comparison group, pre-intervention characteristics were not adequately described, and statistical analyses were not optimised.

Table 2: Quality Appraisal and Characteristics of Studies

\begin{tabular}{|c|c|c|c|c|c|}
\hline Author: year & Study design & Country & Industry/sector & Sample size & $\begin{array}{l}\text { Methodological } \\
\text { quality }\end{array}$ \\
\hline $\begin{array}{l}\text { Aaras: } \\
\text { 1999, 2001, } 2002\end{array}$ & $\begin{array}{l}\text { Non-randomised } \\
\text { prospective } \\
\text { parallel group }\end{array}$ & Norway & Computer workers & $\begin{array}{l}\mathrm{l}=32 \\
\mathrm{C}=35\end{array}$ & Medium \\
\hline $\begin{array}{l}\text { Andersen, L: } \\
\text { 2011, 2014; } \\
\text { Lidegaard: } 2013\end{array}$ & RCT & Denmark & Office workers & $\begin{array}{l}I 1=66 \\
I 2=66 \\
C=66\end{array}$ & High \\
\hline $\begin{array}{l}\text { Andersen, C: } \\
2011(P), 2014\end{array}$ & RCT & Denmark & Office workers & $\begin{array}{l}\mathrm{I}=24 \\
\mathrm{C}=23\end{array}$ & High \\
\hline $\begin{array}{l}\text { Bernaards: } \\
\text { 2006(P), } 2008\end{array}$ & RCT & $\begin{array}{l}\text { The } \\
\text { Netherlands }\end{array}$ & Computer workers & $\begin{array}{l}I 1=152 \\
I 2=156 \\
C=158\end{array}$ & High \\
\hline Camargo: 2009 & Single group & Brazil & $\begin{array}{l}\text { Assembly line } \\
\text { workers }\end{array}$ & $\mathrm{I}=17$ & Low \\
\hline Cheng: 2007 & RCT & Hong Kong & $\begin{array}{l}\text { Varied, } \\
\text { Medium physical } \\
\text { demand level of } \\
\text { work }\end{array}$ & $\begin{array}{l}\mathrm{I}=51 \\
\mathrm{C}=52\end{array}$ & Medium \\
\hline Dropkin: 2015 & RCT & USA & Computer workers & $\begin{array}{l}\mathrm{I}=56 \\
\mathrm{C}=57\end{array}$ & High \\
\hline $\begin{array}{l}\text { Esmaeilzadeh: } \\
2014\end{array}$ & RCT & Turkey & Computer workers & $\begin{array}{l}\mathrm{I}=47 \\
\mathrm{C}=47\end{array}$ & Medium \\
\hline Feuerstein: 2004 & RCT & USA & $\begin{array}{l}\text { Professional office } \\
\text { workers }\end{array}$ & $\begin{array}{l}11=47 \\
I 2=46\end{array}$ & High \\
\hline Hagberg: 2000 & RCT & Sweden & Industrial workers & $\begin{array}{l}11==43 \\
12=34\end{array}$ & Medium \\
\hline Jay: 2014 & RCT & Denmark & $\begin{array}{l}\text { Laboratory } \\
\text { technicians and } \\
\text { office workers }\end{array}$ & $\begin{array}{l}I 1=19 \\
I 2=19\end{array}$ & High \\
\hline Lincoln: 2002 & RCT & USA & $\begin{array}{l}\text { Varied: Managers, } \\
\text { clerks, postal } \\
\text { carriers, } \\
\text { mechanical/electrical } \\
\text { workers }\end{array}$ & $\begin{array}{l}\mathrm{I}=53 \\
\mathrm{C}=48\end{array}$ & Medium \\
\hline Lundblad: 1999 & RCT & Sweden & Industrial workers & $\begin{array}{l}I 1=32 \\
I 2=33 \\
C=32\end{array}$ & High \\
\hline $\begin{array}{l}\text { Martimo: 2010; } \\
\text { Shiri: } 2011\end{array}$ & RCT & Finland & $\begin{array}{l}\text { Varied: Nurses and } \\
\text { other healthcare } \\
\text { workers, clerical } \\
\text { workers and } \\
\text { secretaries, } \\
\text { warehouse workers }\end{array}$ & $\begin{array}{l}\mathrm{I}=91 \\
\mathrm{C}=86\end{array}$ & Medium \\
\hline Ripat; 2006 & RCT & Canada & Computer workers & $\begin{array}{l}\mathrm{I}=43 \\
\mathrm{C}=25\end{array}$ & Medium \\
\hline $\begin{array}{l}\text { Sundstrup: } \\
\text { 2013(P2006 } \\
\text { ), 2014, 2014, } \\
\text { 2016; Andersen: } \\
\text { 2017 }\end{array}$ & RCT & Denmark & $\begin{array}{l}\text { Slaughterhouse } \\
\text { workers }\end{array}$ & $\begin{array}{l}I=33 \\
C=33\end{array}$ & High \\
\hline Voerman: 2007 & RCT & $\begin{array}{l}\text { Sweden \& } \\
\text { The } \\
\text { Netherlands }\end{array}$ & Computer workers & $\begin{array}{l}\mathrm{I}=42 \\
\mathrm{C}=37\end{array}$ & High \\
\hline
\end{tabular}

$\mathrm{P}=$ Protocol, $\mathrm{I}=$ Intervention group, $\mathrm{C}=$ Control group, $\mathrm{RCT}=$ Randomised controlled trial 


\section{Data extraction}

\section{Characteristics of included studies}

The majority of the included studies were randomised controlled trials $(n=15)$, reported across 23 articles [16-23,23-33,35-42,42]. The remaining two studies were: one of non-randomised prospective parallel group design, reported across three articles [33-35] and one of single group design [43] (see Table 2).

Studies originated in Denmark $(n=4)$ [16-25,31], Sweden $(n=2)[32,38]$, USA $(n=3)[29,30,42]$, Norway $(n=1)$ [33-35], the Netherlands $(n=1)[27,28]$, both Sweden and the Netherlands $(n=1)$ [26], Finland $(n=1)[39,41]$, Hong Kong $(n=1)$ [36], Canada $(n=1)$ [40], Turkey $(n=1)$ [37] and Brazil $(n=1)$ [43].

Study participants included office/computer workers ( $n=9)$ [16-20,26-30,33-35,37,40], assembly line workers in the school supply industry $(n=1)$ [43], industrial workers $(n=2)[32,38]$, slaughterhouse workers $(n=1)$ [21-25], and a combination of laboratory technicians and office workers $(n=1)$ [31]. The remaining three studies had participants with varied occupations; one of these had participants working in the medium category of physical demand [36], the second included managers, clerks, postal carriers and mechanical/electrical workers [42], and the third involved healthcare workers, clerical and warehouse workers [39,41].

Sample sizes varied between 17 and 466 . Attrition also varied greatly, from $0,04 \%$ to $40 \%$. Only two of the studies had attrition rates above $35 \%[32,39,41]$.

\section{Interventions}

Interventions were grouped as far as possible into the following categories: 1) Ergonomic controls $(n=3)[29,33-35,40], 2)$ Ergonomic training and workstation adjustments $(n=4)$ $[27,28,30,37,39,41], 3)$ Exercise/resistance training $(n=6)[16-25,31,38,43]$. Four studies did not match any of these categories and thus will be discussed separately $[26,32,36,42]$ (see Table 3). Outcomes varied across studies, but mostly included symptom severity (notably pain), sick 
leave, postural/ergonomic changes, strength and general measures of health and upper limbrelated disability. 
Table 3: Description of Interventions, Main Outcomes and Findings

\begin{tabular}{|c|c|c|c|c|c|c|c|c|c|}
\hline Authors: year & $\begin{array}{l}\text { Intervention } \\
\text { description }\end{array}$ & $\begin{array}{l}\text { Intervention } \\
\text { provider }\end{array}$ & $\begin{array}{l}\text { Frequency and } \\
\text { duration of } \\
\text { intervention }\end{array}$ & Main outcomes & $\begin{array}{l}\text { Findings: Intervention } \\
\text { group / I1 group }\end{array}$ & $\begin{array}{l}\text { Findings: Control group/ } 12 \\
\text { group }\end{array}$ & $\begin{array}{l}\text { Findings: Between } \\
\text { group comparisons }\end{array}$ & Conclusion & $\begin{array}{l}\text { Length of } \\
\text { observatior }\end{array}$ \\
\hline \multicolumn{10}{|c|}{ Ergonomic controls } \\
\hline \multirow[t]{3}{*}{$\begin{array}{l}\text { Aaras: } \\
\text { 1999, 2001, } \\
2002\end{array}$} & \multirow[t]{3}{*}{$\begin{array}{l}\text { l=Adapted mouse (more } \\
\text { neutral wrist and } \\
\text { forearm position) } \\
\mathrm{C}=\text { Traditional mouse } \\
\text { (pronated forearm) }\end{array}$} & \multirow[t]{3}{*}{ Not Applicable } & \multirow[t]{3}{*}{ Daily use } & Pain & $\begin{array}{l}\text { I: Significant improvements in } \\
\text { wristhand, forearm, shoulder } \\
\text { and neck pain intensity, } \\
\text { frequency and duration at } 6 \\
\text { months, maintained at } 12 \text { and } \\
36 \text { months. }\end{array}$ & $\begin{array}{l}\text { C: No significant } \\
\text { improvement in wrist/hand, } \\
\text { forearm, shoulder and neck } \\
\text { pain intensity, frequency and } \\
\text { duration at } 6 \text { months. Control } \\
\text { group given the intervention } \\
\text { after } 6 \text { months. }\end{array}$ & \multirow[t]{3}{*}{$\begin{array}{l}\text { Significant BG } \\
\text { differences in all } \\
\text { outcomes, in favour of I } \\
\text { group. }\end{array}$} & \multirow[t]{3}{*}{$\begin{array}{l}\text { Anir mouse use showed } \\
\text { overwhelmingly positive } \\
\text { outcomes in terms of } \\
\text { pain, headache, MS sick } \\
\text { leave. }\end{array}$} & \multirow[t]{3}{*}{$\begin{array}{l}6 \text { months } \\
12 \text { months } \\
36 \text { months }\end{array}$} \\
\hline & & & & Headache & $\begin{array}{l}\text { I: Significant improvement in } \\
\text { headache at } 6 \text { months. } \\
\text { Maintained at } 12 \text { and } 36 \\
\text { months. }\end{array}$ & $\begin{array}{l}\text { C: No significant } \\
\text { improvement in headache at } \\
6 \text { months. }\end{array}$ & & & \\
\hline & & & & $\begin{array}{l}\text { Musculoskeletal } \\
\text { sick leave }\end{array}$ & $\begin{array}{l}\text { : Significant decrease in MS } \\
\text { sick leave at } 6 \text { months. } \\
\text { Maintained at } 12 \text { and } 36 \\
\text { months. }\end{array}$ & $\begin{array}{l}\text { C: Increase in MS sick leave } \\
\text { at } 6 \text { months. }\end{array}$ & & & \\
\hline \multirow[t]{3}{*}{ Dropkin: 2015} & \multirow[t]{3}{*}{$\begin{array}{l}\text { l=Adjustable } \\
\text { keyboard/mouse tray } \\
\text { with padded wrist rest } \\
\text { and touch pad, training } \\
\text { on keyboard shortcuts } \\
\text { C=Training on keyboard } \\
\text { shortcuts }\end{array}$} & \multirow[t]{3}{*}{ Unclear } & \multirow[t]{3}{*}{ Daily use } & Pain & $\begin{array}{l}\text { I: Pain severity reduced in all } \\
\text { areas, SS in dominant and } \\
\text { non-dominant proximal UE. }\end{array}$ & $\begin{array}{l}\text { C: Pain severity reduced in } \\
\text { all areas, SS in dominant and } \\
\text { non-dominant proximal UE. }\end{array}$ & $\begin{array}{l}\text { No significant BG } \\
\text { differences in pain } \\
\text { severity, slight protective } \\
\text { effect in dominant side } \\
\text { and increase in pain on } \\
\text { non-dominant side of } \\
\text { intervention group (not } \\
\text { statistically significant). }\end{array}$ & \multirow[t]{3}{*}{$\begin{array}{l}\text { Intervention resulted in } \\
\text { positive changes to } \\
\text { postures on RULA, but } \\
\text { negative changes on } \\
\text { HAL (non-dominant } \\
\text { hand activity). }\end{array}$} & \multirow[t]{3}{*}{7 months } \\
\hline & & & & $\begin{array}{l}\text { Upper limb } \\
\text { postures } \\
\text { (modified RULA) }\end{array}$ & $\begin{array}{l}\text { I: Reduced non-neutral } \\
\text { postures on modified RULA } \\
\text { in } 4 / 5 \text { domains. }\end{array}$ & $\begin{array}{l}\text { C: Increased non-neutral } \\
\text { postures on RULA in the } \\
\text { same } 4 / 5 \text { domains. }\end{array}$ & $\begin{array}{l}\text { Statistically significant } \\
\text { improvement on RULA } \\
\text { in } 2 / 5 \text { domains in favour } \\
\text { of I group. }\end{array}$ & & \\
\hline & & & & $\begin{array}{l}\text { Hand activity } \\
\text { (HAL test) }\end{array}$ & $\begin{array}{l}\text { I: Non-dominant hand activity } \\
\text { increased (HAL test). }\end{array}$ & $\begin{array}{l}\text { C: No significant changes in } \\
\text { hand activity. }\end{array}$ & $\begin{array}{l}\text { Non-dominant hand } \\
\text { activity increased in I } \\
\text { group (significant), no } \\
\text { other significant BG } \\
\text { findings in hand activity } \\
\text { level. }\end{array}$ & & \\
\hline \multirow[t]{2}{*}{ Ripat; 2006} & $\begin{array}{l}\text { I=Adapted Microsoft } \\
\text { Natural keyboards } \\
\text { (reduced activation } \\
\text { force) }\end{array}$ & Not applicable & Daily use & $\begin{array}{l}\text { Symptom Severity } \\
\text { Scale (SSS) }\end{array}$ & $\begin{array}{l}\text { I: Significant improvement on } \\
\text { SSS at } 12 \text { weeks, maintained } \\
\text { at } 24 \text { weeks. }\end{array}$ & $\begin{array}{l}\text { C: Significant improvement } \\
\text { on SSS at } 12 \text { weeks, } \\
\text { maintained at } 24 \text { weeks. }\end{array}$ & \multirow{2}{*}{$\begin{array}{l}\text { Similar patterns of } \\
\text { reduction in symptom } \\
\text { severity and } \\
\text { improvement in } \\
\text { functional status in I and } \\
\text { C groups. } \\
\text { Non-significant trends } \\
\text { towards improved } \\
\text { function in I group. }\end{array}$} & \multirow{2}{*}{$\begin{array}{l}\text { Positive results in } \\
\text { symptoms and function } \\
\text { found with both } \\
\text { keyboards. Trend } \\
\text { towards improved } \\
\text { function with light-touch } \\
\text { keyboard (not SS). }\end{array}$} & \multirow[t]{2}{*}{24 weeks } \\
\hline & $\begin{array}{l}\text { C=Unadapted Microsoft } \\
\text { Natural keyboards }\end{array}$ & & & $\begin{array}{l}\text { Functional Status } \\
\text { Scale (FSS) }\end{array}$ & $\begin{array}{l}\text { I: Significant improvement on } \\
\text { FSS at } 12 \text { weeks. }\end{array}$ & $\begin{array}{l}\text { C: Significant improvement } \\
\text { on FSS at } 12 \text { weeks. }\end{array}$ & & & \\
\hline \multicolumn{10}{|c|}{ Ergonomic training and workstation adjustments } \\
\hline \multirow[t]{3}{*}{$\begin{array}{l}\text { Bernaards: } \\
2006,2008\end{array}$} & $\begin{array}{l}\text { l1=Work style } \\
\text { (ergonomics, stress) } \\
\text { behaviour counselling }\end{array}$ & Trained counsellor & $\begin{array}{l}11 \text { and } 12=6 \\
\text { group meetings } \\
\text { in a 6-month } \\
\text { neriod }\end{array}$ & $\begin{array}{l}\text { Body posture \& } \\
\text { workstation } \\
\text { adjustment }\end{array}$ & $\begin{array}{l}\text { 11: Significant improvements } \\
\text { on } 5 / 14 \text { items. }\end{array}$ & $\begin{array}{l}\text { 12: Significant improvements } \\
\text { 4/14 items. }\end{array}$ & \multirow{3}{*}{$\begin{array}{l}\text { Both I1 and I2 groups } \\
\text { showed significant } \\
\text { improvements in some } \\
\text { elements of body } \\
\text { posture and workstation } \\
\text { adjustment, and use of } \\
\text { breaks, over the } \\
\text { control/usual care group. } \\
\text { Comparisons between } \\
11 \text { and I2 were not } \\
\text { reported. }\end{array}$} & \multirow{3}{*}{$\begin{array}{l}\text { Work style intervention } \\
\text { had some positive } \\
\text { impact on body posture, } \\
\text { WS adjustment and use } \\
\text { of rest breaks. No effect } \\
\text { on work stress. }\end{array}$} & \multirow[t]{3}{*}{12 months } \\
\hline & \multirow[t]{2}{*}{$\begin{array}{l}\text { I2=Work style and } \\
\text { physical activity } \\
\text { counselling } \\
\text { C=Usual care }\end{array}$} & & & Use of breaks & $\begin{array}{l}\text { 11: Significant improvement in } \\
\text { use of breaks software, and } \\
\text { taking breaks after every } \\
\text { hour of computer work. }\end{array}$ & $\begin{array}{l}\text { 12: Significant improvement in } \\
\text { use of breaks software, use } \\
\text { of short breaks, and changing } \\
\text { position or taking breaks after } \\
\text { every hour of computer work. }\end{array}$ & & & \\
\hline & & & & Work stress & 11: No significant change. & 12: No significant change. & & & \\
\hline
\end{tabular}




\begin{tabular}{|c|c|c|c|c|c|c|c|c|c|}
\hline Authors: year & $\begin{array}{l}\text { Intervention } \\
\text { description }\end{array}$ & $\begin{array}{l}\text { Intervention } \\
\text { provider }\end{array}$ & $\begin{array}{l}\text { Frequency and } \\
\text { duration of } \\
\text { intervention }\end{array}$ & Main outcomes & $\begin{array}{l}\text { Findings: Intervention } \\
\text { group / / group }\end{array}$ & $\begin{array}{l}\text { Findings: Control group/ } 12 \\
\text { group }\end{array}$ & $\begin{array}{l}\text { Findings: Between } \\
\text { group comparisons }\end{array}$ & Conclusion & $\begin{array}{l}\text { Length of } \\
\text { observation }\end{array}$ \\
\hline \multirow[t]{3}{*}{$\begin{array}{l}\text { Esmaeilzadeh: } \\
2014\end{array}$} & \multirow[t]{3}{*}{$\begin{array}{l}\text { I=Ergonomic training, } \\
\text { ergonomic brochure, } \\
\text { workstation adjustment } \\
\text { C=No intervention }\end{array}$} & \multirow[t]{3}{*}{$\begin{array}{l}\text { Physiotherapists } \\
\text { with ergonomics } \\
\text { training }\end{array}$} & \multirow{3}{*}{$\begin{array}{l}2 \times 90-\text { minute } \\
\text { training sessions, } \\
\text { training brochure, } \\
\text { monthly } \\
\text { workstation } \\
\text { evaluations and } \\
\text { adjustments }\end{array}$} & $\begin{array}{l}\text { Ergonomic } \\
\text { exposure } \\
\text { (Ergonomic } \\
\text { Questionnaire) }\end{array}$ & $\begin{array}{l}\text { I: Self-reported postural } \\
\text { abnormalities and improper } \\
\text { equipment locations } \\
\text { significantly decreased. }\end{array}$ & $\begin{array}{l}\text { C: Self-reported postural } \\
\text { abnormalities and improper } \\
\text { equipment locations } \\
\text { increased (not significant). }\end{array}$ & $\begin{array}{l}\text { Significant BG } \\
\text { differences in postural } \\
\text { abnormalities and } \\
\text { improper equipment } \\
\text { locations, in favour of I } \\
\text { group. }\end{array}$ & \multirow[t]{3}{*}{$\begin{array}{l}\text { Multi-component } \\
\text { ergonomic intervention } \\
\text { had a positive effect on } \\
\text { ergonomic risk factors } \\
\text { and musculoskeletal } \\
\text { symptoms. }\end{array}$} & \multirow[t]{3}{*}{6 months } \\
\hline & & & & $\begin{array}{l}\text { Musculoskeletal } \\
\text { symptoms } \\
\text { (Modified NMQ, } \\
\text { VAS) }\end{array}$ & $\begin{array}{l}\text { I: Intensity, duration and } \\
\text { frequency of symptoms } \\
\text { decreased significantly. }\end{array}$ & $\begin{array}{l}\text { C: Intensity of symptoms } \\
\text { increased significantly. No } \\
\text { significant change in duration } \\
\text { and frequency of symptoms. }\end{array}$ & $\begin{array}{l}\text { Statistically significant } \\
\text { BG differences in } \\
\text { intensity, duration and } \\
\text { frequency of symptoms, } \\
\text { in favour of I group. }\end{array}$ & & \\
\hline & & & & $\begin{array}{l}\text { Medical care, } \\
\text { medication use }\end{array}$ & $\begin{array}{l}\text { I: No significant change, } \\
\text { tendency to decrease. }\end{array}$ & C: No significant change. & $\begin{array}{l}\text { No significant BG } \\
\text { differences. }\end{array}$ & & \\
\hline \multirow[t]{3}{*}{$\begin{array}{l}\text { Feuerstein: } \\
2004\end{array}$} & \multirow{3}{*}{$\begin{array}{l}1=\text { Ergonomic } \\
\text { workstation assessment } \\
\text { and adjustments, } \\
\text { stretching exercises. } \\
\text { I2=As above + job } \\
\text { stress management } \\
\text { training }\end{array}$} & \multirow[t]{3}{*}{$\begin{array}{l}\text { Occupational } \\
\text { Health Nurse and } \\
\text { Rehabilitation } \\
\text { Engineer }\end{array}$} & \multirow{3}{*}{$\begin{array}{l}11 \text { \& } 12: \\
\text { Workstation } \\
\text { adjustments at } \\
\text { start, } 3 \text { months } \\
\text { and } 12 \text { months. } \\
12: 2 \times 70 \text { min } \\
\text { stress } \\
\text { management } \\
\text { meetings }\end{array}$} & $\begin{array}{l}\text { Symptoms (Pain - } \\
\text { VAS, DASH } \\
\text { symptom severity) }\end{array}$ & $\begin{array}{l}\text { 11: Pain and DASH symptom } \\
\text { severity significantly } \\
\text { decreased at } 3 \text { months, } \\
\text { maintained at } 12 \text { months. }\end{array}$ & $\begin{array}{l}\text { 12: Pain and DASH symptom } \\
\text { severity significantly } \\
\text { decreased at } 3 \text { months, } \\
\text { maintained at } 12 \text { months. }\end{array}$ & $\begin{array}{l}\text { No significant BG } \\
\text { differences in pain and } \\
\text { symptom severity. }\end{array}$ & \multirow{3}{*}{$\begin{array}{l}\text { Ergonomic workstation } \\
\text { adjustments with } \\
\text { stretching exercises had } \\
\text { a positive effect on pain, } \\
\text { ergonomic risk and work } \\
\text { stress. The addditional } \\
\text { job stress management } \\
\text { training did not } \\
\text { significantly enhance } \\
\text { these effects. }\end{array}$} & \multirow[t]{3}{*}{12 months } \\
\hline & & & & $\begin{array}{l}\text { Ergonomic risk } \\
\text { assessment }\end{array}$ & $\begin{array}{l}\text { 11: Significant improvements } \\
\text { at } 3 \text { months, maintained at } 12 \\
\text { months. }\end{array}$ & $\begin{array}{l}\text { 12: Significant improvements } \\
\text { at } 3 \text { months, maintained at } 12 \\
\text { months. }\end{array}$ & $\begin{array}{l}\text { No significant BG } \\
\text { differences. }\end{array}$ & & \\
\hline & & & & Work stress & $\begin{array}{l}\text { 11: Significant improvements } \\
\text { at } 3 \text { months, maintained at } 12 \\
\text { months. }\end{array}$ & $\begin{array}{l}\text { 12: Significant improvements } \\
\text { at } 3 \text { months, maintained at } 12 \\
\text { months. }\end{array}$ & $\begin{array}{l}\text { No significant BG } \\
\text { differences. }\end{array}$ & & \\
\hline \multirow[t]{3}{*}{$\begin{array}{l}\text { Martimo: } \\
\text { 2010; Shiri: } \\
\text { 2011 }\end{array}$} & \multirow{3}{*}{$\begin{array}{l}\text { I=Usual care plus work } \\
\text { visit by a physiotherapist } \\
\text { (suggested and } \\
\text { negotiated ergonomic } \\
\text { improvements with } \\
\text { employee and } \\
\text { supervisor) } \\
\text { C=Usual care by an } \\
\text { occupational health } \\
\text { doctor }\end{array}$} & \multirow[t]{3}{*}{$\begin{array}{l}\text { I=Physiotherapist } \\
\mathrm{C}=\text { Occupational } \\
\text { health doctor }\end{array}$} & \multirow[t]{3}{*}{ I=One work visit } & $\begin{array}{l}\text { Productivity loss at } \\
\text { work (self- } \\
\text { assessed) }\end{array}$ & $\begin{array}{l}\text { I: Proportion and magnitude } \\
\text { of productivity loss lower at } \\
12 \text { weeks. }\end{array}$ & $\begin{array}{l}\text { C: Proportion and magnitude } \\
\text { of productivity loss higher at } \\
12 \text { weeks. }\end{array}$ & $\begin{array}{l}\text { Statistically significant } \\
\text { BG differences in } \\
\text { proportion and } \\
\text { magnitude of } \\
\text { productivity loss, in } \\
\text { favour of I group. }\end{array}$ & \multirow{3}{*}{$\begin{array}{l}\text { Ergonomic intervention } \\
\text { had a positive effect on } \\
\text { productivity at } 12 \text { weeks } \\
\text { No significant effect on } \\
\text { pain intensity and pain } \\
\text { interference with work } \\
\text { as well as sickness } \\
\text { absence at } 12 \text { weeks } \\
\text { and } 12 \text { months. }\end{array}$} & \multirow[t]{3}{*}{$\begin{array}{l}2 \text { weeks } \\
12 \text { months }\end{array}$} \\
\hline & & & & $\begin{array}{l}\text { Pain intensity and } \\
\text { pain interference } \\
\text { with work }\end{array}$ & $\begin{array}{l}\text { I: Significant decrease over } \\
\text { time (12 weeks and } 12 \\
\text { months). }\end{array}$ & $\begin{array}{l}\text { C: Significant decrease over } \\
\text { time (12 weeks and } 12 \\
\text { months). }\end{array}$ & $\begin{array}{l}\text { No significant BG } \\
\text { differences, tendencies } \\
\text { in favour of I group at } 12 \\
\text { weeks and } 12 \text { months, } \\
\text { especially for pain } \\
\text { interference with work. }\end{array}$ & & \\
\hline & & & & Sickness absence & $\begin{array}{l}\text { I: Similar percentage of } \\
\text { participants with sickness } \\
\text { absence due to UED at } 3 \\
\text { months and } 12 \text { months. }\end{array}$ & $\begin{array}{l}\text { C: Higher percentage of } \\
\text { participants with sickness } \\
\text { absence due to UED at } 12 \\
\text { months. }\end{array}$ & $\begin{array}{l}\text { No significant BG } \\
\text { differences. }\end{array}$ & & \\
\hline \multirow{4}{*}{$\begin{array}{l}\text { Exercise / Res } \\
\text { Andersen, L: } \\
\text { 2011, 2014; } \\
\text { Lidegaard: } \\
2013\end{array}$} & tance training & & & & & & & & \\
\hline & $\begin{array}{l}\text { I1=2-minute PRT with } \\
\text { elastic tubing }\end{array}$ & Physiotherapists & $\begin{array}{l}5 \text { sessions per } \\
\text { week (total of } 10- \\
60 \text { minutes) for }\end{array}$ & Pain & $\begin{array}{l}\text { 11: Significant decrease in } \\
\text { neck/shoulder pain intensity. }\end{array}$ & $\begin{array}{l}\text { 12: Significant decrease in } \\
\text { neck/shoulder pain intensity. }\end{array}$ & \multirow{3}{*}{$\begin{array}{l}\text { No significant } \\
\text { differences between I1 } \\
\text { and I2 in pain intensity, } \\
\text { tenderness and muscle } \\
\text { strength. Both groups } \\
\text { showed significant } \\
\text { improvement over } \\
\text { control group in all } 3 \\
\text { outcomes. }\end{array}$} & \multirow{3}{*}{$\begin{array}{l}\text { Strength training had a } \\
\text { positive effect on pain, } \\
\text { tenderness and muscle } \\
\text { strength. No significant } \\
\text { difference between 2- } \\
\text { min and 12-min training. }\end{array}$} & \multirow[t]{3}{*}{10 weeks } \\
\hline & $\begin{array}{l}\text { I2=12-minute PRT with } \\
\text { elastic tubing }\end{array}$ & & 10 weeks & Tenderness & $\begin{array}{l}\text { 11: Significant decrease in } \\
\text { neck/shoulder tenderness. }\end{array}$ & $\begin{array}{l}\text { 12: Significant decrease in } \\
\text { neck/shoulder tenderness. }\end{array}$ & & & \\
\hline & $\begin{array}{l}\text { C=Weekly email with } \\
\text { general health } \\
\text { information }\end{array}$ & & & Muscle strength & $\begin{array}{l}\text { 11: Significant increase in } \\
\text { muscle strength. }\end{array}$ & $\begin{array}{l}\text { 12: Significant increase in } \\
\text { muscle strength. }\end{array}$ & & & \\
\hline \multirow[t]{2}{*}{$\begin{array}{l}\text { Andersen, C: } \\
2011,2014\end{array}$} & \multirow{2}{*}{$\begin{array}{l}\text { I=Shoulder function } \\
\text { exercises } \\
\text { C=Advised to stay } \\
\text { physically active }\end{array}$} & \multirow[t]{2}{*}{ Instructors } & \multirow{2}{*}{$\begin{array}{l}3 \times 20 \text {-minute } \\
\text { sessions per } \\
\text { week for } 10 \\
\text { weeks }\end{array}$} & Pain & I: Pain intensity decreased. & $\begin{array}{l}\text { C: Slight increase in pain } \\
\text { intensity. }\end{array}$ & $\begin{array}{l}\text { Significant BG difference } \\
\text { in pain intensity, in } \\
\text { favour of I group. }\end{array}$ & \multirow{2}{*}{$\begin{array}{l}\text { Shoulder function } \\
\text { exercises had a positive } \\
\text { effect on pain and } \\
\text { shoulder elevation } \\
\text { strength. }\end{array}$} & \multirow[t]{2}{*}{10 weeks } \\
\hline & & & & Muscle strength & $\begin{array}{l}\text { 1: Shoulder elevation and } \\
\text { scapula protraction strength } \\
\text { increased. }\end{array}$ & $\begin{array}{l}\text { C: Unclear - only BG } \\
\text { differences reported. }\end{array}$ & $\begin{array}{l}\text { Significant BG difference } \\
\text { in shoulder elevation } \\
\text { strength, in favour of I } \\
\text { group. No significant BG } \\
\text { difference in scapula } \\
\text { protraction strength, } \\
\text { tendency in favour of I } \\
\text { group. }\end{array}$ & & \\
\hline
\end{tabular}


Stellenbosch University https://scholar.sun.ac.za

\begin{tabular}{|c|c|c|c|c|c|c|c|c|c|}
\hline Authors: year & $\begin{array}{l}\text { Intervention } \\
\text { description }\end{array}$ & $\begin{array}{l}\text { Intervention } \\
\text { provider }\end{array}$ & $\begin{array}{l}\text { Frequency and } \\
\text { duration of } \\
\text { intervention }\end{array}$ & Main outcomes & $\begin{array}{l}\text { Findings: Intervention } \\
\text { group / li group }\end{array}$ & $\begin{array}{l}\text { Findings: Control group/ } 12 \\
\text { group }\end{array}$ & $\begin{array}{l}\text { Findings: Between } \\
\text { group comparisons }\end{array}$ & Conclusion & $\begin{array}{l}\text { Length of } \\
\text { observation }\end{array}$ \\
\hline \multirow[t]{2}{*}{$\begin{array}{l}\text { Camargo: } \\
2009\end{array}$} & \multirow[t]{2}{*}{$\begin{array}{l}\text { l=Cryotherapy, } \\
\text { stretching and } \\
\text { strengthening exercises }\end{array}$} & \multirow[t]{2}{*}{ Physiotherapist } & \multirow[t]{2}{*}{$\begin{array}{l}\text { Twice weekly for } \\
8 \text { weeks }\end{array}$} & $\begin{array}{l}\text { Upper limb function } \\
\text { (DASH) }\end{array}$ & $\begin{array}{l}\text { I: Significant improvement in } \\
\text { DASH overall and work } \\
\text { module scores. }\end{array}$ & \multirow[t]{2}{*}{ No C group. } & \multirow[t]{2}{*}{ Not applicable. } & \multirow{2}{*}{$\begin{array}{l}\text { Igroup (Cryotherapy, } \\
\text { stretching and } \\
\text { strengthening exercises) } \\
\text { showed positive results } \\
\text { in terms of pain and } \\
\text { upper limb function. No } \\
\text { C group in study. }\end{array}$} & \multirow[t]{2}{*}{8 weeks } \\
\hline & & & & $\begin{array}{l}\text { Pain (McGill Pain } \\
\text { Questionnaire) }\end{array}$ & $\begin{array}{l}\text { 1: Significant decrease in pain } \\
\text { intensity. }\end{array}$ & & & & \\
\hline \multirow[t]{3}{*}{$\begin{array}{l}\text { Hagberg: } \\
2000\end{array}$} & \multirow[t]{3}{*}{$\begin{array}{l}\text { I1=Isometric shoulder } \\
\text { endurance training } \\
\text { 12=Isometric shoulder } \\
\text { strength training }\end{array}$} & \multirow[t]{3}{*}{$\begin{array}{l}\text { Physiotherapists } \\
\text { and home program }\end{array}$} & \multirow[t]{3}{*}{$\begin{array}{l}3 \text { times per week } \\
\text { for } 12 \text { weeks }\end{array}$} & Pain (VAS) & 11: Decrease in pain intensity. & 12: Decrease in pain intensity. & $\begin{array}{l}\text { No significant BG } \\
\text { differences. }\end{array}$ & \multirow{3}{*}{$\begin{array}{l}\text { Isometric shoulder } \\
\text { endurance and shoulder } \\
\text { strength exercises had a a } \\
\text { positive effect on pain, } \\
\text { muscle strength and } \\
\text { endurance. } \\
\text { No significant difference } \\
\text { between endurance and } \\
\text { strength training. } \\
\text { No control group. }\end{array}$} & \multirow[t]{3}{*}{24 weeks } \\
\hline & & & & Muscle strength & $\begin{array}{l}\text { 11: Significant increase in } \\
\text { shoulder muscle strength. } \\
\text { No significant effect on grip } \\
\text { strength. }\end{array}$ & $\begin{array}{l}\text { 12: Significant increase in } \\
\text { shoulder muscle strength. } \\
\text { No significant effect on grip } \\
\text { strength. }\end{array}$ & $\begin{array}{l}\text { No significant BG } \\
\text { differences. }\end{array}$ & & \\
\hline & & & & Endurance & $\begin{array}{l}\text { 11: Significant improvement in } \\
\text { shoulder forward flexion } \\
\text { endurance. }\end{array}$ & $\begin{array}{l}\text { 12: Significant improvement in } \\
\text { shoulder forward flexion } \\
\text { endurance. }\end{array}$ & $\begin{array}{l}\text { No significant BG } \\
\text { differences. }\end{array}$ & & \\
\hline \multirow[t]{2}{*}{ Jay: 2014} & $\begin{array}{l}\text { Elastic tubing exercises } \\
11=4 \text { short instructional } \\
\text { videos with audio and } \\
\text { written instructions with } \\
\text { pictures }\end{array}$ & \multirow[t]{2}{*}{ Trainer } & \multirow[t]{2}{*}{$\begin{array}{l}\text { 12: Up to } 10 \\
\text { minutes, } 5 \text { days } \\
\text { per week }\end{array}$} & $\begin{array}{l}\text { Errors in exercise } \\
\text { execution }\end{array}$ & Not applicable. & Not applicable. & $\begin{array}{l}\text { No significant BG } \\
\text { differences in } 3 / 4 \\
\text { exercises. } \\
\text { Higher error score in } 11 \\
\text { for unilateral shoulder } \\
\text { external rotation. }\end{array}$ & \multirow[t]{2}{*}{$\begin{array}{l}\text { Video-based instruction } \\
\text { and personalised } \\
\text { instruction resulted in } \\
\text { similar performance of } \\
\text { exercises and similar } \\
\text { pain improvement. }\end{array}$} & \multirow[t]{2}{*}{2 weeks } \\
\hline & $\begin{array}{l}\text { I2=As above + option to } \\
\text { attend personalised } \\
\text { instruction sessions as } \\
\text { needed. }\end{array}$ & & & Pain & $\begin{array}{l}\text { 11: Decrease in pain intensity } \\
\text { at } 2 \text { weeks. }\end{array}$ & $\begin{array}{l}\text { 12: Decrease in pain intensity } \\
\text { at } 2 \text { weeks. }\end{array}$ & $\begin{array}{l}\text { No significant BG } \\
\text { differences. }\end{array}$ & & \\
\hline \multirow{7}{*}{$\begin{array}{l}\text { Sundstrup: } \\
\text { 2013, 2014, } \\
\text { 2014, 2016; } \\
\text { Andersen: } \\
2017\end{array}$} & \multirow[t]{7}{*}{$\begin{array}{l}\text { I=Strength training } \\
\mathrm{C}=\text { Participatory } \\
\text { ergonomics }\end{array}$} & \multirow[t]{7}{*}{$\begin{array}{l}\text { I=Training instructor } \\
\mathrm{C}=\text { Ergonomists }\end{array}$} & \multirow[t]{7}{*}{$\begin{array}{l}l=3 \times 10 \text { minutes } \\
\text { per week }\end{array}$} & Pain (VAS) & $\begin{array}{l}\text { I: Decrease in pain intensity } \\
\text { at } 10 \text { weeks. }\end{array}$ & $\begin{array}{l}\text { C: Decrease in pain intensity } \\
\text { at } 10 \text { weeks. }\end{array}$ & $\begin{array}{l}\text { Significant BG difference } \\
\text { in pain intensity, in } \\
\text { favour of I group. }\end{array}$ & \multirow{7}{*}{$\begin{array}{l}\text { Strength training was } \\
\text { more effective than } \\
\text { ergonomic training at } \\
\text { reducing pain and work } \\
\text { disability, increasing } \\
\text { muscle strength and } \\
\text { maintaining work ability. } \\
\text { No significant impact on } \\
\text { mental health, with } \\
\text { moderate positive effect } \\
\text { on vitality and social } \\
\text { climate. }\end{array}$} & \multirow[t]{7}{*}{10 weeks } \\
\hline & & & & $\begin{array}{l}\text { Work disability } \\
\text { (DASH work } \\
\text { module) }\end{array}$ & $\begin{array}{l}\text { I: Work disability reduced at } \\
10 \text { weeks. }\end{array}$ & $\begin{array}{l}\text { C: Work disability increased } \\
\text { at } 10 \text { weeks. }\end{array}$ & $\begin{array}{l}\text { Significant BG difference } \\
\text { in work disability, in } \\
\text { favour of I group. }\end{array}$ & & \\
\hline & & & & Muscle strength & $\begin{array}{l}\text { I: Shoulder rotation and wrist } \\
\text { extension strength increased } \\
\text { at } 10 \text { weeks. }\end{array}$ & $\begin{array}{l}\text { C: Shoulder rotation and wrist } \\
\text { extension strength decreased } \\
\text { at } 10 \text { weeks. }\end{array}$ & $\begin{array}{l}\text { Significant BG difference } \\
\text { in muscle strength, in } \\
\text { favour of I group. }\end{array}$ & & \\
\hline & & & & $\begin{array}{l}\text { Work ability index } \\
\text { (WAI) }\end{array}$ & $\begin{array}{l}\text { I: No significant change in } \\
\text { WAl at } 10 \text { weeks. }\end{array}$ & $\begin{array}{l}\text { C: Significant decrease in } \\
\text { WAl at } 10 \text { weeks. }\end{array}$ & $\begin{array}{l}\text { Significant BG difference } \\
\text { in work ability, in favour } \\
\text { of I group. }\end{array}$ & & \\
\hline & & & & $\begin{array}{l}\text { Mental health } \\
\text { (SF-36) }\end{array}$ & $\begin{array}{l}\text { I: Decline in mental health at } \\
10 \text { weeks. }\end{array}$ & $\begin{array}{l}\text { C: Improvement in mental } \\
\text { health at } 10 \text { weeks. }\end{array}$ & $\begin{array}{l}\text { No significant BG } \\
\text { differences. }\end{array}$ & & \\
\hline & & & & $\begin{array}{l}\text { Social climate (QPS } \\
\text { Nordic) }\end{array}$ & $\begin{array}{l}\text { I: Social climate improved at } \\
\text { 10 weeks }\end{array}$ & $\begin{array}{l}\text { C: Social climate deteriorated } \\
\text { at } 10 \text { weeks. }\end{array}$ & $\begin{array}{l}\text { Significant BG difference } \\
\text { in favour of I group, } \\
\text { moderate effect size. }\end{array}$ & & \\
\hline & & & & Vitality (SF-36) & $\begin{array}{l}\text { I: Vitality improved at } 10 \\
\text { weeks }\end{array}$ & $\begin{array}{l}\text { C: Vitality decreased at } 10 \\
\text { weeks. }\end{array}$ & $\begin{array}{l}\text { Significant BG difference } \\
\text { in favour of I group, } \\
\text { moderate effect size. }\end{array}$ & & \\
\hline
\end{tabular}


Stellenbosch University https://scholar.sun.ac.za

\begin{tabular}{|c|c|c|c|c|c|c|c|c|c|}
\hline Authors: year & $\begin{array}{l}\text { Intervention } \\
\text { description }\end{array}$ & $\begin{array}{l}\text { Intervention } \\
\text { provider }\end{array}$ & $\begin{array}{l}\text { Frequency and } \\
\text { duration of } \\
\text { intervention }\end{array}$ & Main outcomes & $\begin{array}{l}\text { Findings: Intervention } \\
\text { group / I group }\end{array}$ & $\begin{array}{l}\text { Findings: Control group/ } 12 \\
\text { group }\end{array}$ & $\begin{array}{l}\text { Findings: Between } \\
\text { group comparisons }\end{array}$ & Conclusion & $\begin{array}{l}\text { Length of } \\
\text { observation }\end{array}$ \\
\hline \multicolumn{10}{|c|}{ Clinic-based VS Workplace-based Work hardening } \\
\hline \multirow[t]{3}{*}{ Cheng: 2007} & \multirow{3}{*}{$\begin{array}{l}\mathrm{I}=\text { Workplace-based } \\
\text { work hardening training } \\
\mathrm{C}=\text { =Clinic-based work } \\
\text { hardening training }\end{array}$} & \multirow[t]{3}{*}{ Unclear } & \multirow[t]{3}{*}{$\begin{array}{l}3 \text { sessions per } \\
\text { week for } 4 \text { weeks }\end{array}$} & $\begin{array}{l}\text { Self-perceived } \\
\text { shoulder pain and } \\
\text { disability (SPADI) }\end{array}$ & $\begin{array}{l}\text { I: Decrease in SPADI at } 4 \\
\text { weeks. }\end{array}$ & $\begin{array}{l}\text { C: Decrease in SPADI at } 4 \\
\text { weeks. }\end{array}$ & $\begin{array}{l}\text { Significant BG difference } \\
\text { in SPADI, in favour of I } \\
\text { group. }\end{array}$ & \multirow{3}{*}{$\begin{array}{l}\text { Workplace-based work } \\
\text { hardening had a } \\
\text { significantly higher } \\
\text { positive effect on } \\
\text { shoulder pain and } \\
\text { disability, FCE and } \\
\text { return to work. }\end{array}$} & \multirow[t]{3}{*}{4 weeks } \\
\hline & & & & FCE & $\begin{array}{l}\text { I: Improvement in FCE at } 4 \\
\text { weeks. }\end{array}$ & $\begin{array}{l}\text { C: Improvement in FCE at } 4 \\
\text { weeks }\end{array}$ & $\begin{array}{l}\text { Significant BG } \\
\text { differences in shoulder } \\
\text { flexion, arm lifting force, } \\
\text { high-near lifting force, } \\
\text { carrying force, overhead } \\
\text { tolerance; in favour of I } \\
\text { group. }\end{array}$ & & \\
\hline & & & & Return to work & $\begin{array}{l}\text { 1: } 72 \% \text { of workers returned to } \\
\text { normal or modified duties. }\end{array}$ & $\begin{array}{l}\text { C: } 38 \% \text { of workers returned } \\
\text { to normal or modified duties. }\end{array}$ & $\begin{array}{l}\text { Significant BG difference } \\
\text { in favour of I group. }\end{array}$ & & \\
\hline \multicolumn{10}{|c|}{ Nurse case manager training } \\
\hline Lincoln: 2002 & $\begin{array}{l}\text { I=Training program for } \\
\text { nurse case managers } \\
\text { on ergonomic } \\
\text { assessment, worksite } \\
\text { accommodations and } \\
\text { problem-solving. } \\
\text { C=Usual care - Nurse } \\
\text { case managers without } \\
\text { the additional ergonomic } \\
\text { training }\end{array}$ & Unclear & $\begin{array}{l}\text { I=Once-off } \\
\text { training for } 2 \\
\text { days, training } \\
\text { manual, option to } \\
\text { contact } \\
\text { instructors for } \\
\text { further support }\end{array}$ & $\begin{array}{l}\text { Recommended } \\
\text { accommodations }\end{array}$ & $\begin{array}{l}\text { I: Variety in type of } \\
\text { accommodations } \\
\text { recommended (workstation } \\
\text { layout, computer -related } \\
\text { improvements, funishings, } \\
\text { accessories, lifting/carrying } \\
\text { aids). }\end{array}$ & $\begin{array}{l}\text { C: Mostly administrative } \\
\text { accommodations } \\
\text { recommended (lifting } \\
\text { restrictions, modified or light } \\
\text { duty, increased work breaks). }\end{array}$ & $\begin{array}{l}\text { Significant BG } \\
\text { differences number and } \\
\text { type of accommodations } \\
\text { recommended and } \\
\text { implemented, in favour } \\
\text { of I group. } \\
\text { Implementation rates } \\
\text { similar between groups. }\end{array}$ & $\begin{array}{l}\text { Trained nurses had } \\
\text { more accommodations } \\
\text { recommended and } \\
\text { implemented. }\end{array}$ & $\begin{array}{l}\text { Observed unti } \\
\text { completion of } \\
\text { case } \\
\text { management } \\
\text { (varying time } \\
\text { periods) }\end{array}$ \\
\hline \multicolumn{10}{|c|}{ Physiotherapy VS Feldenkrais } \\
\hline \multirow[t]{2}{*}{$\begin{array}{l}\text { Lundblad: } \\
1999\end{array}$} & \multirow[t]{3}{*}{$\begin{array}{l}11=\text { Group-based } \\
\text { physiotherapy } \\
12=\text { Group-based and } \\
\text { individual Feldenkrais } \\
C=\text { No intervention }\end{array}$} & \multirow[t]{2}{*}{$\begin{array}{l}\text { 11=Physiotherapists } \\
\text { |2=Unclear }\end{array}$} & \multirow[t]{2}{*}{$\begin{array}{l}11=50 \text { minutes } \\
\text { twice weekkly for } \\
16 \text { weeks. } \\
12=50 \text { minutes } \\
\text { per week: } 4 \\
\text { individual and } 12 \\
\text { group sessions }\end{array}$} & $\begin{array}{l}\text { Symptoms: } \\
\text { Neck complaints } \\
\text { Shoulder } \\
\text { complaints } \\
\text { Neck Index } \\
\text { Shoulder Index } \\
\text { Neck-Shoulder } \\
\text { Index } \\
\text { Usual pain } \\
\text { Worst pain }\end{array}$ & $\begin{array}{l}\text { 11: No significant changes in } \\
\text { any symptom-outcomes. }\end{array}$ & $\begin{array}{l}\text { 12: Significant improvements } \\
\text { in neck complaints, shoulder } \\
\text { complaints, Neck-Shoulder } \\
\text { Index and usual pain } \\
\text { intensity. No significant } \\
\text { change in Neck Index, } \\
\text { shoulder index and worst } \\
\text { pain. }\end{array}$ & $\begin{array}{l}\text { Significant differences } \\
\text { between I1 and } 12 \text { in } \\
\text { Neck Index, Neck- } \\
\text { Shoulder Index, in } \\
\text { favour of } 12 . \\
\text { Significant decrease in } \\
\text { usual pain in } 12 \text { and C } \\
\text { groups, most } \\
\text { pronounced in } 12 \text { group. }\end{array}$ & \multirow[t]{2}{*}{$\begin{array}{l}\text { Feldenkrais had a } \\
\text { positive effect on neck } \\
\text { complaints, neck- } \\
\text { shoulder complaints and } \\
\text { usual pain intensity. } \\
\text { Physiotherapy had no } \\
\text { significant effect. }\end{array}$} & \multirow[t]{2}{*}{12 months } \\
\hline & & & & Disability & $\begin{array}{l}\text { 11: No significant changes in } \\
\text { sick leave (tendency to } \\
\text { decrease) or disability during } \\
\text { work. }\end{array}$ & $\begin{array}{l}\text { 12: No significant changes in } \\
\text { sick leave of disability during } \\
\text { work (both showed tendency } \\
\text { to decrease). }\end{array}$ & $\begin{array}{l}\text { No significant BG } \\
\text { differences. } 12 \text { showed a } \\
\text { tendency to decrease } \\
\text { and C showed a } \\
\text { tendency to increase } \\
\text { sick leave. }\end{array}$ & & \\
\hline \multicolumn{9}{|c|}{ Ambulant Myofeedback Training } & \\
\hline \multirow[t]{2}{*}{$\begin{array}{l}\text { Voerman: } \\
2007\end{array}$} & $\begin{array}{l}\text { I=Ambulant } \\
\text { myofeedback training } \\
\text { and ergonomic training }\end{array}$ & $\begin{array}{l}\text { Physiotherapist and } \\
\text { health scientists }\end{array}$ & $\begin{array}{l}\mathrm{l}=8 \text { hours per } \\
\text { week for } 4 \\
\text { weeks, plus }\end{array}$ & Pain (VAS) & $\begin{array}{l}\text { I: Significant decrease in } \\
\text { neck/shoulder pain intensity. }\end{array}$ & $\begin{array}{l}\text { C: Significant decrease in } \\
\text { neck/shoulder pain intensity. }\end{array}$ & $\begin{array}{l}\text { No significant BG } \\
\text { differences. }\end{array}$ & \multirow{2}{*}{$\begin{array}{l}\text { Both ergonomic } \\
\text { counselling and } \\
\text { ambulant mofeeedback } \\
\text { had positive effects on } \\
\text { pain and disability. No } \\
\text { significant diffiterences in } \\
\text { effects between the } \\
\text { interventions. }\end{array}$} & \multirow[t]{2}{*}{6 months } \\
\hline & $\begin{array}{l}\mathrm{C}=\text { Ergonomic training } \\
\text { only }\end{array}$ & & $\begin{array}{l}\text { weekly visits by } \\
\text { therapist } \\
C=4 \text { weekly visits } \\
\text { by therapist }\end{array}$ & Pain disability index & $\begin{array}{l}\text { I: Significant decrease in } \\
\text { disability levels. }\end{array}$ & $\begin{array}{l}\text { C: Significant decrease in } \\
\text { disability levels. }\end{array}$ & $\begin{array}{l}\text { No significant BG } \\
\text { differences. }\end{array}$ & & \\
\hline
\end{tabular}

I=Intervention group, C=Control group, BG=Between Group, MS=Musculoskeletal, RULA=Rapid Upper Limb Assessment, HAL=Hand Activity Level, UE=Upper extremity, SSS=Symptom Severity Scale, FSS=Functional Status Scale, SS=Statistically significant, APB=Abductor Pollicis Brevis, NMQ=Nordic Musculoskeletal Questionnaire, VAS=Visual Analogue Scale, UEFS=Upper Extremity Functional Scale, UED=Upper extremity disorders. DASH=Disabilities of the Arm, Shoulder and Hand questionnaire, PRT=Progressive
Resistance Training, RPE=Rating of Perceived Exertion, WAI=Work Ability Index, SPADI=Shoulder Pain and Disability Index, QPS Nordic - General Nordic Questionnaire for psychological and social factors at work, FCE=Functional Capacity Evaluation, ROM=Range Of Motion 


\section{Ergonomic controls}

The ergonomic controls investigated included a non-traditional mouse, which uses a more neutral wrist and forearm position [33-35]; an adjustable keyboard-mouse tray with a touch pad in the non-dominant hand [29]; and reduced-force keyboards [40]. One of these studies was rated as high quality [29], and the remaining two studies were of medium methodological quality [33-35,40]. Results of the Aaras study [33-35], which assessed outcomes at 6 months, 12 months and 36 months, suggest that use of a mouse enabling more neutral forearm and wrist position compared with a standard mouse may reduce pain, headache and musculoskeletal-related sick leave. The Dropkin study [29], which was of high quality, found that while some ergonomic postures improved with the intervention, hand activity in the distal non-dominant arm increased, possibly due to the 11 functions of the touch pad. The Ripat study [40] results were complicated by the use of a Microsoft Naturals keyboard in both intervention and control groups, while the intervention group's keyboards were adapted to also reduce activation force. Improvements were seen in symptom severity and functional status of both groups, with no significant between-group differences.

\section{Ergonomic training and Workstation adjustments}

Four studies were included in this category, of which two were high quality $[27,28,30]$ and two medium quality $[37,39,41]$. Workstation adjustments were conducted by physiotherapists in two of the studies $[37,39,41]$. Alternately, interventions were offered by occupational health nurses, rehabilitation engineers and counsellors [27,28,30]. The Feuerstein study [30], which was of high quality, offered workstation adjustment to the control group as well, and found that improvement in outcomes were seen in both groups, with no significant between-group differences. Similarly, the high quality Bernaards $[27,28]$ study offered the same work style behaviour counselling to two intervention groups, while the second intervention group also received physical activity counselling. Both intervention groups showed significant improvements over a control group, which received usual care, but direct comparisons between the two intervention groups were not reported. The Esmaeilzadeh study [37], which 
offered no control intervention, found that ergonomic postures and musculoskeletal symptoms improved significantly more in the intervention group than the control group. The Martimo/Shiri study found that productivity loss was significantly lower in the intervention compared to the control group [39,41].

\section{Exercise / Resistance training}

This was the largest intervention category, with six studies included across 13 articles. Four of these studies were high quality [16-25,31], with one medium quality study [38] and one low quality study (no control group) [43]. Three of the studies offered the intervention through physiotherapists [18-20,38,43], and in the remaining three studies the intervention providers were described as trainers or instructors [16,17,21-25,31]. Results of all studies were positive, with improvements seen in pain [16-25,31,38,43], strength [16-25,38], functional ability [2125,43], work ability [21-25,43], absenteeism [38] and medication use [38] in intervention groups. The Andersen/Lidegaard study [16-18] found that as little as two minutes of resistance training five times per week had a marked positive impact on pain, tenderness and muscle strength. Jay et al [31] assessed errors in exercise execution between an intervention group using video-based training and a control group using personalised instruction, and found that the two groups had similar error scores, training frequency and pain improvements.

\section{Clinic-based Vs Workplace-based Work hardening}

The Cheng study [36], based in Hong Kong, investigated the effect of a workplace-based work hardening program on workers with work-related rotator cuff tendonitis. This was a medium quality study. A job coach contacted the worksites to arrange for the workers' actual work tasks to be used as treatment media. A control group of conventional clinic-based work hardening was used. It was found that the intervention group had significantly higher improvements on the functional capacity evaluation, lower Shoulder Pain and Disability Index (SPADI) scores, and a higher percentage of the group successfully returned to work. 


\section{Nurse case manager training}

An RCT of medium quality, based in the USA, involved training randomly selected nurse case managers in Integrated Case Management for two days [42]. The training program included ergonomic assessment, worksite accommodations and problem-solving. The nurses' approach to case managing workers with WRUEDs was then compared with usual care (nurses who had not undergone the specialised training) in the same population. The intervention group was found to make more recommendations, with more variety in the types of accommodations recommended, compared with the control group. Trained nurses also had a higher number of accommodations implemented, although implementation rates were the same between intervention and control groups.

\section{Physiotherapy Vs Feldenkrais}

One high quality study investigated the difference between group-based physiotherapy and Feldenkrais interventions amongst female industrial workers at an automotive factory with neck/shoulder complaints [32]. Interventions took the form of 50-minute weekly sessions for 16 weeks. Feldenkrais interventions aimed to increase sensory awareness, investigate common movement and postural patterns, break stereotyped movement patterns, and enable self-care for neck, shoulder and back complaints through guided movement sequences [32]. Physiotherapy sessions aimed to increase knowledge of body and pain, learn back stabilising exercises and improve postural awareness by practicing work-related lift and movement techniques in a group exercise program. The Feldenkrais group showed a significant decrease in neck/shoulder complaints, compared with the physiotherapy and control groups, while no significant changes were found in any outcomes in the physiotherapy group.

\section{Ambulant Myofeedback training}

Voerman et al [26], in a high quality study, investigated the effect of a myofeedback system with harness worn by participants for 4 weeks. The device assessed and recorded muscle activity in the upper trapezius, and provided sound and vibration feedback to participants at intervals, prompting them to relax. Both intervention and control groups also received 
ergonomic counselling, with weekly visits from their therapists. Both groups experienced significant improvements in pain and disability, with no significant difference between groups. 


\section{Discussion}

This systematic review aimed to determine the effectiveness of workplace-based rehabilitative interventions in workers with upper limb conditions on work performance, pain, absenteeism, productivity and other outcomes. Twenty-eight suitable articles were found, which were grouped into 17 studies and seven intervention categories (see Table 3). The largest body of evidence was found to support workplace exercise programs, of which four out of six were high quality studies. Positive effects were also found for use of ergonomic controls, ergonomic training and workstation adjustments, although these intervention categories had fewer high quality studies. The remaining intervention categories (work hardening, myofeedback training, Feldenkrais, nurse case manager training) only had one study each. While results of three of these studies were encouraging, with interventions showing significant positive effects, recommendations for practice should be made with caution as there are only one medium or high quality study per intervention.

Four high quality studies [16-25,31], one medium quality study [38] and one low quality study [43] supported workplace exercise programs. The research suggests that these programs may be effective whether including strength or endurance training programs [38], using as little as two minutes of regular exercise [16-18], or basing them on high quality video instruction rather than a personalised trainer [31]. In the case of ergonomic controls, it is difficult to draw conclusions. Studies finding that an adapted mouse (Anir mouse) significantly reduced pain, headaches and musculoskeletal sick leave at 6 months and were maintained at 12 and 36 months, were of medium quality [33-35]; while keyboard adjustments and shortcuts had mixed results $[29,40]$. The two high quality studies on workstation adjustments $[27,28,30]$ offered ergonomic training or workstation adjustment to both intervention groups, although the Bernaards study $[27,28]$ also included a control group which received usual care. In the Feuerstein study [30], both intervention groups showed significant improvements, but there were no significant between group differences. Inclusion of a third (control) group that did not 
receive any workstation adjustment or ergonomic training, as in the Bernaards study $[27,28]$, may have more clearly highlighted the effects of the intervention. The remaining two studies on workstation adjustment and ergonomic training $[37,39,41]$ found statistically significant improvements in use of breaks and some elements of body posture, as well as productivity loss at work.

The Cheng study [36] supports work hardening that has a workplace-based component, rather than clinic-based work hardening. While the workplace-based group showed significantly better outcomes than the clinic-based group, the authors cautioned that the process of change is not well understood: the improvements in intervention group could be due to a number of factors, including the presence of the job coach or the provision of modified work duties. Further study in smaller organisations and considering longer term effects was recommended.

Feldenkrais was found to be more effective than physiotherapy in the Lundblad study [32]. The authors postulated that this could be because Feldenkrais has a stronger focus on participants' perceptions and experiences than physiotherapy, and that Feldenkrais has some features in common with relaxation and biofeedback techniques, which have been found to be effective at reducing pain [32].

In the Voerman study [26], the ambulant myofeedback training group did not have significantly different outcomes to the control group. The researchers attributed this to the presence of subgroups in which the intervention was more beneficial (e.g. workers with certain cognitivebehavioural characteristics), the use of too generic outcomes (pain and disability were used instead of outcomes related to the specific working mechanisms of the intervention), and the low initial pain and disability levels, resulting in a smaller potential for improvement.

As expected, the present review identified more relevant studies than the 2004 review on workplace-based rehabilitative interventions with work related upper extremity disorders [4], which included eight studies. Intervention categories were similar across the two reviews. The Williams review [4] concluded that there was insufficient evidence to identify and make 
recommendations regarding effective workplace-based rehabilitative interventions for upper limb conditions. Our conclusions regarding the one study common to both reviews [42] are similar, but we are able to make more recommendations regarding workplace exercise, ergonomic controls and adjustments due to research published subsequent to the 2004 review.

Six studies $[26,30,32,34,40,41]$ included in our review were also included in the reviews on preventative workplace interventions with upper limb conditions [2,11], as these reviews included secondary and tertiary preventative interventions which also qualify as rehabilitative interventions. Quality assessment and data extraction matched between the reviews. One more study [36] was also included in a recent IWH review on workplace-based return-to-work interventions for musculoskeletal, pain-related and mental health conditions [44]. The authors of this review similarly concluded that there was insufficient evidence to draw conclusions and make recommendations regarding the effectiveness of work hardening programs, as too few high and medium quality studies with positive results were available. 


\section{Strengths and Limitations of this review}

A meta-analysis could not be conducted in this review, due to the heterogeneity of outcomes. This is consistent with other reviews in the field $[2,4,11,44]$. Instead, a critical analysis and narrative synthesis was provided, in order to provide practitioners with the opportunity to draw from studies most relevant to their needs, at varying levels of evidence.

The quality appraisal of studies was optimised through inclusion of all articles related to the studies, e.g. study protocols. This enabled access to all published information on the study methodology, which is not always available in articles reporting primary outcomes.

Publication bias was not addressed, as grey literature was not included. This means that studies with positive results were more likely to be included in our review. We attempted to be as inclusive as possible by seeking expert advice, utilising a wide range of search terms and including multiple databases in our literature search. We also included all languages in our initial search, only excluding one article at full text review stage due to difficulty translating. In spite of this, our search did not yield all of the studies expected. Notably, seven of the eight studies included in the Williams review [4] were not retrieved in our literature search, despite using more databases, a wider inclusion criteria (all upper limb conditions were included, rather than only WRUEDs) and searching the reference lists of included articles for further studies. One of the databases used in the Williams review (EMBASE) could not be included in our review, as we did not have access to this database through the library service of Stellenbosch University. It may also be related to changes in database content and MeSH terms over time, differences in use of Boolean operators between the two reviews or the structure of our search strategy. It is thus possible that not all relevant studies were found in our review. 


\section{Recommendations for future research}

Several of the included studies showed positive effects with no significant between-group results, likely due to insufficient difference between intervention and control group. It is recommended that when two differing interventions are studied, an additional control group is included, which may be more likely to highlight intervention effects.

Quality appraisal of included studies was hampered by unclear reporting or inadequate statistical analyses. Future studies should clearly report on the details of and participation in intervention, examine and adjust for pre-intervention differences, examine for differences between dropout and remaining participants, perform intention-to-treat analyses, and always perform direct between-group comparisons. It is also recommended that a follow-up assessment after 3 months or more is included in the study, to assess long term effects of the intervention. These factors will reduce the risk of bias in individual studies and enable clearer interpretation of results.

Most studies included pain as an outcome, assessed through the Visual Analogue Scale (VAS). More standardised reporting may have enabled meta-analyses in different categories of intervention. The continued use of outcome measures such as the VAS, DASH, UEFS and RULA is encouraged, to ensure valid and reliable data and improve the potential for homogeneity between studies.

In future studies, researchers should pre-determine the minimum effect size that would demonstrate clinical significance for participants, as statistical significance is not necessarily indicative of clinical significance [45].

\section{Recommendations for practice}

The use of workplace exercise programs in rehabilitation of upper limb conditions is well supported by the evidence. Clinicians may consider implementing strength or endurance training programs of regular, short duration. Larger populations of workers may be reached 
through use of high quality instructional videos. Group programs appear to be effective at reducing upper limb symptoms and improving function.

Workstation adjustment and ergonomic training appear to be beneficial in reducing ergonomic risk, improving musculoskeletal symptoms and productivity. Job stress management training had no significant effect and is therefore not recommended.

Ergonomic controls vary significantly and should therefore be evaluated by their individual merit. An adapted computer mouse enabling more neutral wrist and forearm postures may be beneficial in reducing upper limb pain, headaches and musculoskeletal sick leave, with the effect maintained at 12 and 36 months. Adjustable keyboard/mouse trays and ergonomically adapted keyboards may be beneficial, but care should be taken to assess whether these adaptations shift hand activity or non-neutral postures to the non-dominant hand.

Workplace-based work hardening, case manager training and Feldenkrais should be implemented with caution, as only one study supported each of these interventions. Ambulant myofeedback training is not recommended. 


\section{Conclusions}

High quality evidence was found in favour of workplace exercise programs in a variety of employment settings. Positive effects included reduced pain, increased muscle strength and endurance, maintenance of work ability, improved upper limb function and reduction in work disability. Mixed evidence was found for ergonomic controls: medium quality evidence with strong positive results for an adapted mouse using more neutral forearm and wrist positions; mixed results for an adjustable keyboard-mouse tray with touch pad in the non-dominant hand; and positive effects for Microsoft Naturals keyboards, with no significant improvement with use of reduced force keyboards. Workplace adjustments, ergonomic training and work style behaviour counselling also showed positive effects, while job stress management training had no significant additional effect. Positive effects were seen for workplace-based work hardening, Feldenkrais and case manager training, but more research needs to be conducted on these interventions. Ambulant myofeedback training had no significant effect compared with ergonomic training. 


\section{Acknowledgements}

None.

\section{Compliance with Ethical Standards}

\section{Funding}

No funding was obtained for this study.

\section{Conflict of interest}

The authors declare that they have no conflict of interest.

\section{Informed consent}

This article does not contain any studies with human participants or animals performed by any of the authors. 


\section{References}

[1] Schultz G, Mostert K, Rothmann I. Repetitive strain injury among South African employees: The relationship with burnout and work engagement. International Journal of Industrial Ergonomics. 2012 Sep 30;42(5):449-56.

[2] Van Eerd D, Munhall C, Irvin E, Rempel D, Brewer S, Van Der Beek AJ, Dennerlein JT, Tullar J, Skivington K, Pinion C, Amick B. Effectiveness of workplace interventions in the prevention of upper extremity musculoskeletal disorders and symptoms: an update of the evidence. Occup Environ Med. 2016 Jan 1;73(1):62-70.

[3] Kielkowski D, Rees D, Bradshaw D. Burden of occupational morbidity in South Africa: Two large field surveys of self-reported work-related and work-aggravated disease. South African journal of science. 2004 Jul 1;100(7-8):399-402.

[4] Williams RM, Westmorland MG, Schmuck G, MacDermid JC. Effectiveness of workplace rehabilitation interventions in the treatment of work-related upper extremity disorders: a systematic review. Journal of Hand Therapy. 2004 Jun 30;17(2):267-73.

[5] Edries $\mathrm{N}$, Jelsma $\mathrm{J}$, Maart $\mathrm{S}$. The impact of an employee wellness programme in clothing/textile manufacturing companies: a randomised controlled trial. BMC public health. 2013 Jan 11;13(1):25.

[6] Franche RL, Cullen K, Clarke J, Irvin E, Sinclair S, Frank J, Institute for Work \& Health (IWH) Workplace-Based RTW Intervention Literature Review Research Team. Workplacebased return-to-work interventions: a systematic review of the quantitative literature. Journal of occupational rehabilitation. 2005 Dec 1;15(4):607-31.

[7] Schiller J. Bringing in treatment: Establishing a workplace rehabilitation program. Risk Management. 1996 May 1;43(5):49. 
[8] Cancelliere C, Donovan J, Stochkendahl MJ, Biscardi M, Ammendolia C, Myburgh C, Cassidy JD. Factors affecting return to work after injury or illness: best evidence synthesis of systematic reviews. Chiropractic \& manual therapies. 2016 Dec 1;24(1):32.

[9] Burns PB, Rohrich RJ, Chung KC. The levels of evidence and their role in evidence-based medicine. Plastic and reconstructive surgery. 2011 Jul;128(1):305.

[10] MacDermid JC. An introduction to evidence-based practice for hand therapists. Journal of Hand Therapy. 2004 Jun 30;17(2):105-17.

[11] Kennedy CA, Amick III BC, Dennerlein JT, Brewer S, Catli S, Williams R, Serra C, Gerr F, Irvin E, Mahood Q, Franzblau A. Systematic review of the role of occupational health and safety interventions in the prevention of upper extremity musculoskeletal symptoms, signs, disorders, injuries, claims and lost time. Journal of occupational rehabilitation. 2010 Jun $1 ; 20(2): 127-62$

[12] Moher D, Shamseer L, Clarke M, Ghersi D, Liberati A, Petticrew M, Shekelle P, Stewart LA. Preferred reporting items for systematic review and meta-analysis protocols (PRISMA-P) 2015 statement. Syst rev. 2015 Jan 1;4(1):1.

[13] Shamseer L, Moher D, Clarke M, Ghersi D, Liberati A, Petticrew M, Shekelle P, Stewart LA. Preferred reporting items for systematic review and meta-analysis protocols (PRISMA-P) 2015: elaboration and explanation. Bmj. 2015 Jan 2;349:g7647.

[14] Liberati A, Altman DG, Tetzlaff J, Mulrow C, Gøtzsche PC, loannidis JP, Clarke M, Devereaux PJ, Kleijnen J, Moher D. The PRISMA statement for reporting systematic reviews and meta-analyses of studies that evaluate health care interventions: explanation and elaboration. PLoS medicine. 2009 Jul 21;6(7):e1000100.

[15] American Occupational Therapy Association. Guidelines for Systematic Reviews. 2014:19. 
[16] Andersen LL, Saervoll CA, Mortensen OS, Poulsen OM, Hannerz H, Zebis MK. Effectiveness of small daily amounts of progressive resistance training for frequent neck/shoulder pain: randomised controlled trial. PAIN®. 2011 Feb 28;152(2):440-6.

[17] Andersen LL, Zebis MK. Process evaluation of workplace interventions with physical exercise to reduce musculoskeletal disorders. International journal of rheumatology. 2014 Dec $10 ; 2014$.

[18] Lidegard M, Jensen RB, Andersen CH, Zebis MK, Colado JC, Wang Y, Heilskov-Hansen T, Andersen LL. Effect of brief daily resistance training on occupational neck/shoulder muscle activity in office workers with chronic pain: randomized controlled trial. BioMed research international. 2013 Dec 31;2013.

[19] Andersen CH, Sjøgaard G, Andersen LL, Zebis MK, Mortensen OS. Protocol for shoulder function training reducing musculoskeletal pain in shoulder and neck: a randomized controlled trial. BMC musculoskeletal disorders. $2011 \mathrm{Dec} ; 12(1): 14$.

[20] Andersen CH, Andersen LL, Zebis MK, Sjøgaard G. Effect of scapular function training on chronic pain in the neck/shoulder region: a randomized controlled trial. Journal of occupational rehabilitation. 2014 Jun 1;24(2):316-24.

[21] Andersen LL, Persson R, Jakobsen MD, Sundstrup E. Psychosocial effects of workplace physical exercise among workers with chronic pain: Randomized controlled trial. Medicine. 2017 Jan;96(1).

[22] Sundstrup E, Jakobsen MD, Andersen CH, Jay K, Persson R, Aagaard P, Andersen LL. Participatory ergonomic intervention versus strength training on chronic pain and work disability in slaughterhouse workers: study protocol for a single-blind, randomized controlled trial. BMC musculoskeletal disorders. 2013 Feb 21;14(1):67.

[23] Sundstrup E, Jakobsen MD, Brandt M, Jay K, Persson R, Aagaard P, Andersen LL. Workplace strength training prevents deterioration of work ability among workers with chronic 
pain and work disability: a randomized controlled trial. Scandinavian journal of work, environment \& health. 2014 May 1:244-51.

[24] Sundstrup E, Jakobsen MD, Andersen CH, Jay K, Persson R, Aagaard P, Andersen LL. Effect of two contrasting interventions on upper limb chronic pain and disability: a randomized controlled trial. Pain Physician. 2014 Mar 1;17(2):145-54.

[25] Sundstrup E, Jakobsen MD, Brandt M, Jay K, Aagaard P, Andersen LL. Strength Training Improves Fatigue Resistance and Self-Rated Health in Workers with Chronic Pain: A Randomized Controlled Trial. BioMed research international. 2016 Oct 17;2016.

[26] Voerman GE, Sandsjö L, Vollenbroek-Hutten MM, Larsman P, Kadefors R, Hermens HJ. Effects of ambulant myofeedback training and ergonomic counselling in female computer workers with work-related neck-shoulder complaints: a randomized controlled trial. Journal of occupational rehabilitation. 2007 Mar 1;17(1):137-52.

[27] Bernaards CM, Ariëns GA, Hildebrandt VH. The (cost-) effectiveness of a lifestyle physical activity intervention in addition to a work style intervention on the recovery from neck and upper limb symptoms in computer workers. BMC musculoskeletal disorders. 2006 Oct $24 ; 7(1): 80$.

[28] Bernaards CM, Ariëns GA, Simons M, Knol DL, Hildebrandt VH. Improving work style behavior in computer workers with neck and upper limb symptoms. Journal of occupational rehabilitation. 2008 Mar 1;18(1):87-101

[29] Dropkin J, Kim H, Punnett L, Wegman DH, Warren N, Buchholz B. Effect of an office ergonomic randomised controlled trial among workers with neck and upper extremity pain. Occup Environ Med 2015;72[1]:6.

[30] Feuerstein M, Nicholas RA, Huang GD, Dimberg L, Ali D, Rogers H. Job stress management and ergonomic intervention for work-related upper extremity symptoms. Applied ergonomics. 2004 Nov 30;35(6):565-74. 
[31] Jay K, Brandt M, Andersen LL. Effect of video-based versus personalized instruction on errors during elastic tubing exercises for musculoskeletal pain: a randomized controlled trial. BioMed research international. 2014 Mar 10;2014.

[32] Lundblad I, Elert J, Gerdle B. Randomized controlled trial of physiotherapy and Feldenkrais interventions in female workers with neck-shoulder complaints. Journal of Occupational Rehabilitation. 1999 Sep 1;9(3):179-94.

[33] Aaras A, Ro O, Thoresen M. Can a more neutral position of the forearm when operating a computer mouse reduce the pain level for visual display unit operators? A prospective epidemiological intervention study. International Journal of Human-Computer Interaction. 1999 Jun 1;11(2):79-94.

[34] Aarås A, Dainoff M, Ro O, Thoresen M. Can a more neutral position of the forearm when operating a computer mouse reduce the pain level for visual display unit operators? A prospective epidemiological intervention study: part II. International Journal of HumanComputer Interaction. 2001 Mar 1;13(1):13-40.

[35] Aarås A, Dainoff M, Ro O, Thoresen M. Can a more neutral position of the forearm when operating a computer mouse reduce the pain level for VDU operators? International Journal of Industrial Ergonomics. 2002 Nov 30;30(4):307-24.

[36] Cheng AS, Hung LK. Randomized controlled trial of workplace-based rehabilitation for work-related rotator cuff disorder. Journal of occupational rehabilitation. 2007 Sep $1 ; 17(3): 487-503$.

[37] Esmaeilzadeh S, Ozcan E, Capan N. Effects of ergonomic intervention on work-related upper extremity musculoskeletal disorders among computer workers: a randomized controlled trial. International archives of occupational and environmental health. 2014 Jan 1;87(1):73-83.

[38] Hagberg M, Harms-Ringdahl K, Nisell R, Hjelm EW. Rehabilitation of neck-shoulder pain in women industrial workers: a randomized trial comparing isometric shoulder endurance 
training with isometric shoulder strength training. Archives of physical medicine and rehabilitation. 2000 Aug 31;81(8):1051-8.

[39] Martimo KP, Shiri R, Miranda H, Ketola R, Varonen H, Viikari-Juntura E. Effectiveness of an ergonomic intervention on the productivity of workers with upper-extremity disorders-a randomized controlled trial. Scandinavian journal of work, environment \& health. 2010 Jan 1:25-33.

[40] Ripat J, Scatliff T, Giesbrecht E, Quanbury A, Friesen M, Kelso S. The effect of alternate style keyboards on severity of symptoms and functional status of individuals with work related upper extremity disorders. Journal of occupational rehabilitation. 2006 Dec 1;16(4):707-18.

[41] Shiri R, Martimo KP, Miranda H, Ketola R, Kaila-Kangas L, Liira H, Karppinen J, ViikariJuntura E. The effect of workplace intervention on pain and sickness absence caused by upper-extremity musculoskeletal disorders. Scandinavian journal of work, environment \& health. 2011 Mar 1:120-8.

[42] Lincoln AE, Feuerstein M, Shaw WS, Miller VI. Impact of case manager training on worksite accommodations in workers' compensation claimants with upper extremity disorders. Journal of Occupational and Environmental Medicine. 2002 Mar 1;44(3):237-45.

[43] Camargo PR, Haik MN, Ludewig PM, Filho RB, Mattiello-Rosa SM, Salvini TF. Effects of strengthening and stretching exercises applied during working hours on pain and physical impairment in workers with subacromial impingement syndrome. Physiotherapy theory and practice. 2009 Jan 1;25(7):463-75

[44] Cullen KL, Irvin E, Collie A, Clay F, Gensby U, Jennings PA, Hogg-Johnson S, Kristman V, Laberge M, McKenzie D, Newnam S. Effectiveness of Workplace Interventions in Returnto-Work for Musculoskeletal, Pain-Related and Mental Health Conditions: An Update of the Evidence and Messages for Practitioners. Journal of Occupational Rehabilitation. 2017 Feb $21: 1-5$ 
[45] Burger M, Kriel R, Damon A, Abel A, Bansda A, Wakens M, Ernstzen D. The effectiveness of low-level laser therapy on pain, self-reported hand function, and grip strength compared to placebo or "sham" treatment for adults with carpal tunnel syndrome: A systematic review. Physiotherapy Theory and Practice. 2017 Mar 4;33(3):184-97. 


\section{Chapter 3: Conclusion}

Several systematic reviews have been conducted on workplace-based interventions for musculoskeletal conditions, but the current review is the first which specifically focuses on workplace-based rehabilitative interventions for all upper limb conditions.

The initial literature search yielded 1071 titles (see Addendum B for search strings and detailed results). After title and abstract screening, full text review, and scanning of included articles' reference lists, 17 studies were included, which were published across 28 articles. Quality appraisal of the studies was conducted using the IWH Risk of Bias tool. Nine studies were rated as high quality, seven as medium quality, and one as low quality (see Addendum C for rating scores). Studies were categorised by intervention, as follows:

1. Ergonomic controls $(n=3)$;

2. Ergonomic training and workstation adjustments $(n=4)$;

3. Exercise and resistance training $(n=6)$;

4. Clinic-based vs workplace-based work hardening $(n=1)$;

5. Nurse case manager training $(n=1)$;

6. Physiotherapy vs Feldenkrais $(n=1)$;

7. Ambulant myofeedback training $(n=1)$.

Positive effects were found for workplace exercise and ergonomic training/workstation adjustments. Results were mixed for ergonomic controls. Ambulant myofeedback training showed no effect. The remaining three categories had positive effects, but only one study per category.

Similarly to other reviews, workplace exercise programs were found to have the largest body of high quality research supporting their value in improving upper limb musculoskeletal symptoms and work ability. It is important to note that this does not mean that workplace exercise programs are more effective than other rehabilitation programs, rather that they have 
been better researched. Research on group-based exercise programs may be inherently easier to conduct than more individualised workplace interventions, such as reasonable accommodations, workstation and job task adjustments, splinting, job rotation and alternate placement, where participant numbers will be lower. In addition, all four of the high quality studies on workplace exercise programs were conducted by researchers from the National Research Centre for the Working Environment in Copenhagen, Denmark, who are likely to have resources and support aiding them in conducting high quality research.

Interestingly, only one of the included studies was clearly designed by occupational therapists [13], while several of the intervention programs were conducted by physiotherapists [14-22]. Furthermore, 15 of the 17 included studies were conducted in high income countries, while only two studies were conducted in upper middle income countries similar to South Africa (Turkey and Brazil) $[18,19]$. This raises several questions:

1. Are physiotherapists more likely to be employed in occupational health settings than occupational therapists? One study referred to physiotherapists in the Finnish occupational health services receiving advanced training in occupational health and ergonomics [20].

2. If this is the case, is it prevalent in Northern European or high income countries only? The only study designed by occupational therapists was conducted in Hong Kong [13].

3. Are occupational therapists as active in occupational health care but less likely to conduct and publish research?

Providing answers to these questions is beyond the scope of this study, but certainly does give food for thought, and brings to mind recommendations for future research:

1. Research institutions should endeavour towards diversity in staff, employing rehabilitation professionals with a variety of areas of expertise. This will aid in reducing bias in the types of research conducted and published. 
2. Researchers at research institutions and universities should build partnerships with clinicians in practice, to assist in designing and publishing more high quality research in "real-life" rehabilitation contexts.

3. More research needs to be conducted on individualised rehabilitation. Study protocols could potentially be designed by professional interest groups, such as OTOH (Occupational Therapy in Occupational Health) or WPG (Work Practice Group) in South Africa, in order to collate data from several practitioners or institutions.

4. The gap between research in high income and middle to low income countries needs to be addressed, although this is a difficult problem to overcome, as high income countries would inevitably have more resources for conducting and publishing research. Partnerships through international organisations such as the World Federation of Occupational Therapy (WFOT), the International Labour Office (ILO) or the World Health Organisation (WHO) may be beneficial to this end.

Issues were raised in the introduction of this report, related to the development of workplacebased rehabilitation services in South Africa. The results of the current systematic review, while more relevant to high income countries, do suggest the following recommendations for practice:

1. Collaboration with the Department of Labour to build health resources for work environments, such as those described in the Lincoln study [23], is encouraged. It is acknowledged that this is not without challenges in the South African context, but this process is critical to bridging the gap between legislation and practice.

2. Several of the interventions were conducted in partnership with workers or workplace supervisors [20,23]. This aligns with occupational therapy values, and should be included in design of future programs.

3. Workplace-based rehabilitation programs should consider including an exercise / resistance training component, possibly through collaboration with physiotherapists. 
4. Programs should be designed with anticipated outcomes in mind. Ergonomic controls or training may be more suitable in work environments or with workers requiring reduction in ergonomic risk, while strengthening programs may be more suitable if considerable muscle strength is an inherent requirement of the job.

5. Practitioners should take care to select and use appropriate outcome measures to document the outcomes of interventions, considering the psychometric properties of these measures, particularly as these apply to the South African context.

6. Long term follow-up should be included in rehabilitation programs, in order to determine lasting effects of intervention. Reassessment of outcomes at three to six months after cessation of intervention is recommended.

Recommendations for education:

1. Undergraduate curricula should include training on international and South African legislation and policies related to work disability and rehabilitation, along with exposure to current practices in workplace-based rehabilitation by occupational therapists and other practitioners.

2. Postgraduate training should include education on the setup and provision of workplace-based rehabilitation services; collaboration with key role players such as managers and supervisors, as well as other occupational health staff; addressing challenges specific to the workplace; selecting workplace-specific, responsive outcomes; development of evidence based intervention programs suitable to the workplace; and collection of suitable data for future research.

Workplace-based rehabilitation is a growing field of practice for occupational therapists and other practitioners. It is well supported by legislation. There is a clear need for intervention specific to upper limb conditions in the workplace. While challenges persist in the provision of work rehabilitation services in South Africa, It is hoped that the findings and recommendations of this study will assist in the application of evidence based practice in this area. 


\section{Chapter 4: References}

[1] Kielkowski D, Rees D, Bradshaw D. Burden of occupational morbidity in South Africa: Two large field surveys of self-reported work-related and work-aggravated disease. South African journal of science. 2004 Jul 1;100(7-8):399-402.

[2] Van Eerd D, Munhall C, Irvin E, Rempel D, Brewer S, Van Der Beek AJ, Dennerlein JT, Tullar J, Skivington K, Pinion C, Amick B. Effectiveness of workplace interventions in the prevention of upper extremity musculoskeletal disorders and symptoms: an update of the evidence. Occup Environ Med. 2016 Jan 1;73(1):62-70.

[3] Schultz G, Mostert K, Rothmann I. Repetitive strain injury among South African employees: The relationship with burnout and work engagement. International Journal of Industrial Ergonomics. 2012 Sep 30;42(5):449-56.

[4] Compensation Commissioner R. The Compensation Commissioner's Guidelines for Health Practitioners \& Employers to manage Work-related Upper Limb Disorders. 2004.

[5] United Nations. United Nations Convention on the Rights of Persons with Disabilities. 2006.

[6] International Labour Office. ILO Convention 159 and Recommendation 168. 2008.

[7] South African Government. Integrated National Disability Strategy. 1997.

[8] South African Department of Labour. Compensation Fund Strategic Plan 2014/5 - 2018/9. 2015.

[9] Ver Loren Van Themaat, Dorita Cornelius. The practice profile of occupational therapists delivering work practice services in South Africa. South Africa: University of Cape Town; 2015. [10] Ilott I. Evidence-based Practice: A Critical Appraisal. Occupational therapy international $2012 ; 19[1]: 1-6$. 
[11] Cameron KA, Ballantyne S, Kulbitsky A, Margolis-Gal M, Daugherty T, Ludwig F. Utilization of evidence-based practice by registered occupational therapists. Occupational therapy international. 2005 Sep 1;12(3):123-36.

[12] Buchanan $\mathrm{H}$. The uptake of evidence-based practice by occupational therapists in South Africa. World Federation of Occupational Therapists Bulletin. 2011 Nov 1;64(1):29-38.

[13] Cheng AS, Hung LK. Randomized controlled trial of workplace-based rehabilitation for work-related rotator cuff disorder. Journal of occupational rehabilitation. 2007 Sep $1 ; 17(3): 487-503$

[14] Lundblad I, Elert J, Gerdle B. Randomized controlled trial of physiotherapy and Feldenkrais interventions in female workers with neck-shoulder complaints. Journal of Occupational Rehabilitation. 1999 Sep 1;9(3):179-94.

[15] Lidegard M, Jensen RB, Andersen CH, Zebis MK, Colado JC, Wang Y, Heilskov-Hansen T, Andersen LL. Effect of brief daily resistance training on occupational neck/shoulder muscle activity in office workers with chronic pain: randomized controlled trial. BioMed research international. 2013 Dec 31;2013.

[16] Andersen LL, Saervoll CA, Mortensen OS, Poulsen OM, Hannerz H, Zebis MK. Effectiveness of small daily amounts of progressive resistance training for frequent neck/shoulder pain: randomised controlled trial. PAIN®. 2011 Feb 28;152(2):440-6.

[17] Andersen LL, Zebis MK. Process evaluation of workplace interventions with physical exercise to reduce musculoskeletal disorders. International journal of rheumatology. 2014 Dec $10 ; 2014$.

[18] Camargo PR, Haik MN, Ludewig PM, Filho RB, Mattiello-Rosa SM, Salvini TF. Effects of strengthening and stretching exercises applied during working hours on pain and physical impairment in workers with subacromial impingement syndrome. Physiotherapy theory and practice. 2009 Jan 1;25(7):463-75. 
[19] Esmaeilzadeh S, Ozcan E, Capan N. Effects of ergonomic intervention on work-related upper extremity musculoskeletal disorders among computer workers: a randomized controlled trial. International archives of occupational and environmental health. 2014 Jan 1;87(1):73-83. [20] Martimo KP, Shiri R, Miranda H, Ketola R, Varonen H, Viikari-Juntura E. Effectiveness of an ergonomic intervention on the productivity of workers with upper-extremity disorders-a randomized controlled trial. Scandinavian journal of work, environment \& health. 2010 Jan $1: 25-33$

[21] Voerman GE, Sandsjö L, Vollenbroek-Hutten MM, Larsman P, Kadefors R, Hermens HJ. Effects of ambulant myofeedback training and ergonomic counselling in female computer workers with work-related neck-shoulder complaints: a randomized controlled trial. Journal of occupational rehabilitation. 2007 Mar 1;17(1):137-52.

[22] Shiri R, Martimo KP, Miranda H, Ketola R, Kaila-Kangas L, Liira H, Karppinen J, Viikari-

Juntura $E$. The effect of workplace intervention on pain and sickness absence caused by upper-extremity musculoskeletal disorders. Scandinavian journal of work, environment \& health. 2011 Mar 1:120-8.

[23] Lincoln AE, Feuerstein M, Shaw WS, Miller VI. Impact of case manager training on worksite accommodations in workers' compensation claimants with upper extremity disorders. Journal of Occupational and Environmental Medicine. 2002 Mar 1;44(3):237-45. 


\section{Addendum A: Journal Guidelines}

\section{Journal of Occupational Rehabilitation: Instructions for}

\section{Authors}

\section{TYPES OF PAPERS}

Original Paper, Review, Editorial, Introduction, Book review, Brief communication, News, Report, erratum, etc.

\section{EDITORIAL PROCEDURE}

\section{Single-blind peer review}

This journal follows a single-blind reviewing procedure. Authors are therefore requested to submit a title page, containing title, all author names, affiliations, and the contact information of the corresponding author. Any acknowledgements, disclosures, or funding information should also be included on this page.

\section{MANUSCRIPT SUBMISSION}

\section{Manuscript Submission}

Submission of a manuscript implies: that the work described has not been published before; that it is not under consideration for publication anywhere else; that its publication has been approved by all co-authors, if any, as well as by the responsible authorities - tacitly or explicitly - at the institute where the work has been carried out. The publisher will not be held legally responsible should there be any claims for compensation.

\section{Permissions}

Authors wishing to include figures, tables, or text passages that have already been published elsewhere are required to obtain permission from the copyright owner(s) for both the print and online format and to include evidence that such permission has been granted when submitting 
their papers. Any material received without such evidence will be assumed to originate from the authors.

\section{Online Submission}

Please follow the hyperlink "Submit online" on the right and upload all of your manuscript files following the instructions given on the screen.

\section{TITLE PAGE}

The title page should include:

- The name(s) of the author(s)

- A concise and informative title

- The affiliation(s) and address(es) of the author(s)

- The e-mail address, telephone and fax numbers of the corresponding author

\section{Abstract}

Please provide a structured abstract of 150 to 250 words which should be divided into the following sections:

- Purpose (stating the main purposes and research question)

- Methods

- Results

- Conclusions

\section{Keywords}

Please provide up to 5 key words selected from the National Library of Medicine-Medical Subject Headings (http://www.nlm.nih.gov/MeSH/MBrowser.html).

\section{TEXT}

\section{Text Formatting}

Manuscripts should be submitted in Word.

- Use a normal, plain font (e.g., 10-point Times Roman) for text. 
- Use italics for emphasis.

- Use the automatic page numbering function to number the pages.

- Do not use field functions.

- Use tab stops or other commands for indents, not the space bar.

- Use the table function, not spreadsheets, to make tables.

- Use the equation editor or MathType for equations.

- Save your file in docx format (Word 2007 or higher) or doc format (older Word versions).

Manuscripts with mathematical content can also be submitted in LaTeX.

- LaTeX macro package (zip, $182 \mathrm{kB}$ )

\section{Headings}

Please use no more than three levels of displayed headings.

\section{Abbreviations}

Abbreviations should be defined at first mention and used consistently thereafter.

\section{Footnotes}

Footnotes can be used to give additional information, which may include the citation of a reference included in the reference list. They should not consist solely of a reference citation, and they should never include the bibliographic details of a reference. They should also not contain any figures or tables.

Footnotes to the text are numbered consecutively; those to tables should be indicated by superscript lower-case letters (or asterisks for significance values and other statistical data). Footnotes to the title or the authors of the article are not given reference symbols.

Always use footnotes instead of endnotes. 


\section{Acknowledgments}

Acknowledgments of people, grants, funds, etc. should be placed in a separate section on the title page. The names of funding organizations should be written in full.

As a guide, manuscripts should not normally exceed 5000 words (excluding references and abstract) or have more than 5 tables or figures or more than 50 references (more references may be allowable for systematic reviews)."

Manuscripts are typically 15-20 double-spaced typed pages. Table and figures should be limited to 3-4 total. If you think your article will be significantly shorter or longer than that average, please include an explanation along with your submission.

\section{SCIENTIFIC STYLE}

- Please always use internationally accepted signs and symbols for units (SI units).

- Nomenclature: Insofar as possible, authors should use systematic names similar to those used by Chemical Abstract Service or IUPAC.

- Genus and species names should be in italics.

- Generic names of drugs and pesticides are preferred; if trade names are used, the generic name should be given at first mention.

- Please use the standard mathematical notation for formulae, symbols, etc.:

- Italic for single letters that denote mathematical constants, variables, and unknown quantities

- Roman/upright for numerals, operators, and punctuation, and commonly defined functions or abbreviations, e.g., cos, det, e or exp, lim, log, max, min, sin, tan, $d$ (for derivative)

- Bold for vectors, tensors, and matrices.

Statistical software: Include the name of the manufacturer along with the manufacturer's location after first appearance in text. 


\section{REFERENCES}

\section{Citation}

Reference citations in the text should be identified by numbers in square brackets. Some examples:

1. Negotiation research spans many disciplines [3].

2. This result was later contradicted by Becker and Seligman [5].

3. This effect has been widely studied [1-3, 7].

\section{Reference list}

The list of references should only include works that are cited in the text and that have been published or accepted for publication. Personal communications and unpublished works should only be mentioned in the text. Translate all non-English titles into English. Do not use footnotes or endnotes as a substitute for a reference list.

The entries in the list should be numbered consecutively to follow the order of citations in the text.

\section{Journal article}

Smith JJ. The world of science. Am J Sci. 1999;36(4):234-235.

Article by DOI

Slifka MK, Whitton JL. Clinical implications of dysregulated cytokine production. J Mol Med. 2000; doi:10.1007/s001090000086

\section{Book}

Blenkinsopp A, Paxton P. Symptoms in the pharmacy: a guide to the management of common illness. 3rd ed. Oxford: Blackwell Science; 1998. 


\section{Book chapter}

Wyllie AH, Kerr JFR, Currie AR. Cell death: the significance of apoptosis. In: Bourne GH, Danielli JF, Jeon KW, editors. International review of cytology. London: Academic; 1980. pp. 251-306.

\section{Online document}

Doe J. Title of subordinate document. In: The dictionary of substances and their effects. Royal Society of Chemistry. 1999. http://www.rsc.org/dose/title of subordinate document. Accessed 15 Jan 1999.

Always use the standard abbreviation of a journal's name according to the ISSN List of Title Word Abbreviations.

If you are unsure, please use the full journal title.

\section{TABLES}

- All tables are to be numbered using Arabic numerals.

- Tables should always be cited in text in consecutive numerical order.

- For each table, please supply a table caption (title) explaining the components of the table.

- Identify any previously published material by giving the original source in the form of a reference at the end of the table caption.

- Footnotes to tables should be indicated by superscript lower-case letters (or asterisks for significance values and other statistical data) and included beneath the table body.

\section{ARTWORK AND ILLUSTRATIONS GUIDELINES}

\section{Electronic Figure Submission}

- Supply all figures electronically.

- Indicate what graphics program was used to create the artwork.

- For vector graphics, the preferred format is EPS; for halftones, please use TIFF format. MSOffice files are also acceptable. 
- Vector graphics containing fonts must have the fonts embedded in the files.

- Name your figure files with "Fig" and the figure number, e.g., Fig1.eps.

\section{Line Art}

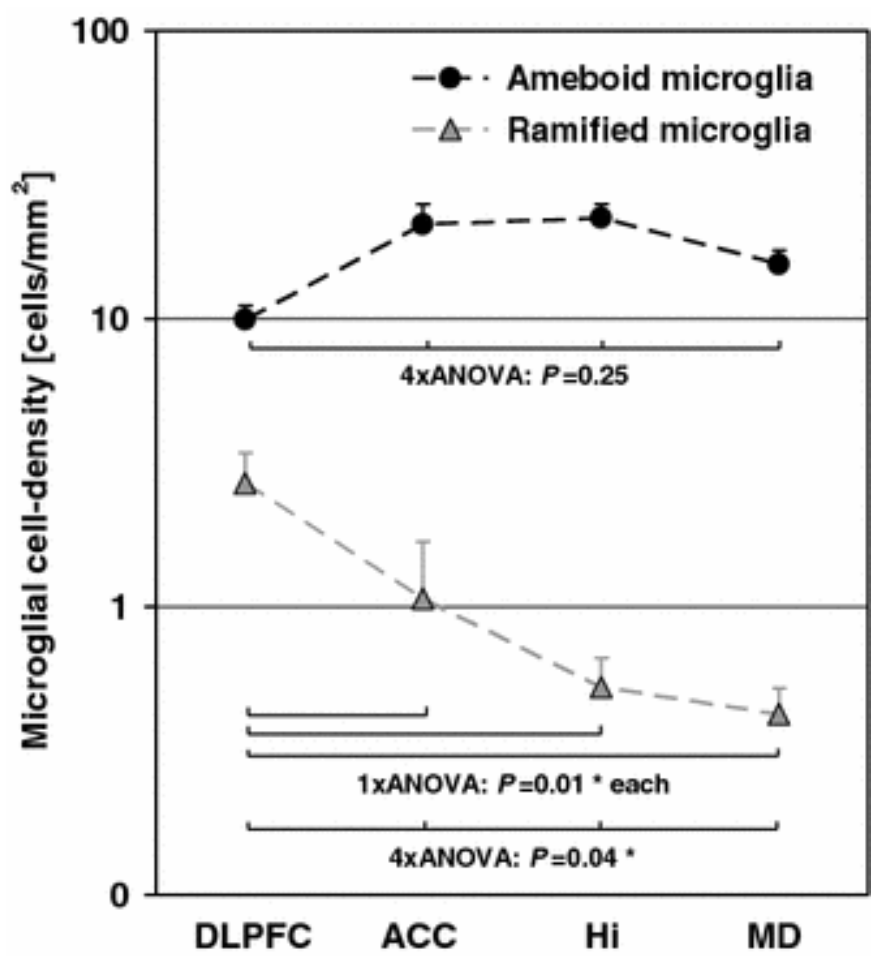

- Definition: Black and white graphic with no shading.

- Do not use faint lines and/or lettering and check that all lines and lettering within the figures are legible at final size.

- All lines should be at least $0.1 \mathrm{~mm}(0.3 \mathrm{pt})$ wide.

- Scanned line drawings and line drawings in bitmap format should have a minimum resolution of $1200 \mathrm{dpi}$.

- Vector graphics containing fonts must have the fonts embedded in the files. 


\section{Halftone Art}

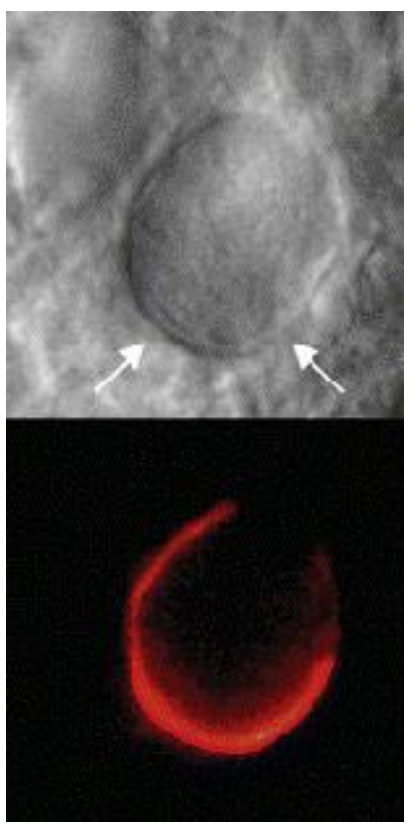

- Definition: Photographs, drawings, or paintings with fine shading, etc.

- If any magnification is used in the photographs, indicate this by using scale bars within the figures themselves.

- Halftones should have a minimum resolution of $300 \mathrm{dpi}$.

\section{Combination Art}

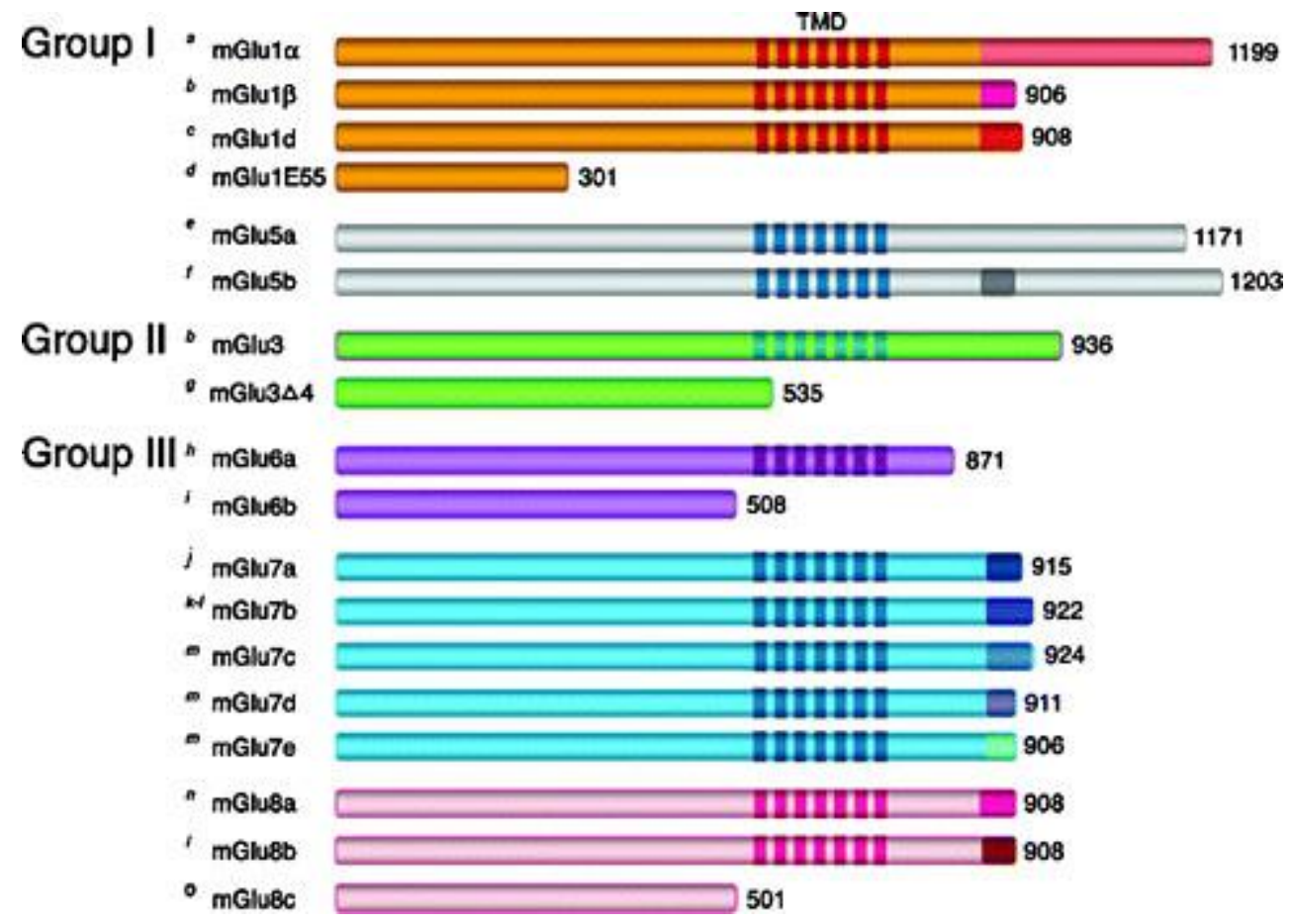


- Definition: a combination of halftone and line art, e.g., halftones containing line drawing, extensive lettering, color diagrams, etc.

- Combination artwork should have a minimum resolution of $600 \mathrm{dpi}$.

\section{Color Art}

- Color art is free of charge for online publication.

- If black and white will be shown in the print version, make sure that the main information will still be visible. Many colors are not distinguishable from one another when converted to black and white. A simple way to check this is to make a xerographic copy to see if the necessary distinctions between the different colors are still apparent.

- If the figures will be printed in black and white, do not refer to color in the captions.

- Color illustrations should be submitted as RGB (8 bits per channel).

\section{Figure Lettering}

- To add lettering, it is best to use Helvetica or Arial (sans serif fonts).

- Keep lettering consistently sized throughout your final-sized artwork, usually about 2$3 \mathrm{~mm}(8-12 \mathrm{pt})$.

- Variance of type size within an illustration should be minimal, e.g., do not use 8-pt type on an axis and 20-pt type for the axis label.

- Avoid effects such as shading, outline letters, etc.

- Do not include titles or captions within your illustrations.

\section{Figure Numbering}

- $\quad$ All figures are to be numbered using Arabic numerals.

- Figures should always be cited in text in consecutive numerical order.

- Figure parts should be denoted by lowercase letters (a, b, c, etc.).

- If an appendix appears in your article and it contains one or more figures, continue the consecutive numbering of the main text. Do not number the appendix figures, "A1, A2, 
A3, etc." Figures in online appendices (Electronic Supplementary Material) should, however, be numbered separately.

\section{Figure Captions}

- Each figure should have a concise caption describing accurately what the figure depicts. Include the captions in the text file of the manuscript, not in the figure file.

- Figure captions begin with the term Fig. in bold type, followed by the figure number, also in bold type.

- No punctuation is to be included after the number, nor is any punctuation to be placed at the end of the caption.

- Identify all elements found in the figure in the figure caption; and use boxes, circles, etc., as coordinate points in graphs.

- Identify previously published material by giving the original source in the form of a reference citation at the end of the figure caption.

\section{Figure Placement and Size}

- Figures should be submitted separately from the text, if possible.

- When preparing your figures, size figures to fit in the column width.

- For most journals the figures should be $39 \mathrm{~mm}, 84 \mathrm{~mm}, 129 \mathrm{~mm}$, or $174 \mathrm{~mm}$ wide and not higher than $234 \mathrm{~mm}$.

- For books and book-sized journals, the figures should be $80 \mathrm{~mm}$ or $122 \mathrm{~mm}$ wide and not higher than $198 \mathrm{~mm}$.

\section{Permissions}

If you include figures that have already been published elsewhere, you must obtain permission from the copyright owner(s) for both the print and online format. Please be aware that some publishers do not grant electronic rights for free and that Springer will not be able to refund any costs that may have occurred to receive these permissions. In such cases, material from other sources should be used. 


\section{Accessibility}

In order to give people of all abilities and disabilities access to the content of your figures, please make sure that

- All figures have descriptive captions (blind users could then use a text-to-speech software or a text-to-Braille hardware)

- Patterns are used instead of or in addition to colors for conveying information (colorblind users would then be able to distinguish the visual elements)

- Any figure lettering has a contrast ratio of at least 4.5:1

\section{ELECTRONIC SUPPLEMENTARY MATERIAL}

Springer accepts electronic multimedia files (animations, movies, audio, etc.) and other supplementary files to be published online along with an article or a book chapter. This feature can add dimension to the author's article, as certain information cannot be printed or is more convenient in electronic form.

Before submitting research datasets as electronic supplementary material, authors should read the journal's Research data policy. We encourage research data to be archived in data repositories wherever possible.

\section{Submission}

- Supply all supplementary material in standard file formats.

- Please include in each file the following information: article title, journal name, author names; affiliation and e-mail address of the corresponding author.

- To accommodate user downloads, please keep in mind that larger-sized files may require very long download times and that some users may experience other problems during downloading.

Audio, Video, and Animations

- Aspect ratio: $16: 9$ or $4: 3$

- Maximum file size: $25 \mathrm{~GB}$ 
- Minimum video duration: $1 \mathrm{sec}$

- Supported file formats: avi, wmv, mp4, mov, m2p, mp2, mpg, mpeg, flv, mxf, mts, m4v, $3 g p$

\section{Text and Presentations}

- Submit your material in PDF format; .doc or .ppt files are not suitable for long-term viability.

- A collection of figures may also be combined in a PDF file.

\section{Spreadsheets}

- Spreadsheets should be submitted as .csv or .xlsx files (MS Excel).

\section{Specialized Formats}

- Specialized format such as .pdb (chemical), .wrl (VRML), .nb (Mathematica notebook), and tex can also be supplied.

\section{Collecting Multiple Files}

- It is possible to collect multiple files in a .zip or .gz file.

\section{Numbering}

- If supplying any supplementary material, the text must make specific mention of the material as a citation, similar to that of figures and tables.

- Refer to the supplementary files as "Online Resource", e.g., "... as shown in the animation (Online Resource 3)", “... additional data are given in Online Resource 4”.

- Name the files consecutively, e.g. "ESM_3.mpg", "ESM_4.pdf".

\section{Captions}

- For each supplementary material, please supply a concise caption describing the content of the file. 


\section{Processing of supplementary files}

- Electronic supplementary material will be published as received from the author without any conversion, editing, or reformatting.

\section{Accessibility}

In order to give people of all abilities and disabilities access to the content of your supplementary files, please make sure that

- The manuscript contains a descriptive caption for each supplementary material

- Video files do not contain anything that flashes more than three times per second (so that users prone to seizures caused by such effects are not put at risk)

\section{ETHICAL STANDARDS}

\section{Conflict of interest}

When authors submit a manuscript, they are responsible for disclosing all financial and personal relationships that might bias their work. To prevent ambiguity, authors must state explicitly whether potential conflicts do or do not exist. Each author must indicate whether or not they have a financial relationship with the organization that sponsored the research. For each source of funds, both the research funder and the grant number should be given.

Conflict of interest statements should be present on every manuscript before the References section. The statement should mention each author separately by name.

Recommended wording is as follows:

Author X declares that he has no conflict of interest.

Author $\mathrm{Y}$ has received research grants from Drug Company $\mathrm{A}$.

Author $\mathrm{Z}$ has received a speaker honorarium from Drug Company B and owns stock in Drug Company C.

If multiple authors declare no conflict, this can be done in one sentence: 
Author $\mathrm{X}$, Author $\mathrm{Y}$ and Author $\mathrm{Z}$ declare that they have no conflict of interest.

The manuscript must also be accompanied by the "Authorship \& Disclosure Form" on the journal website.

Follow the below link for Springer's Conflict of Interest Statement:

http://www.springer.com/authors?SGWID=0-111-6-791531-0

\section{Informed consent}

For studies with human subjects, please include the following statement before the References section:

'All procedures followed were in accordance with the ethical standards of the responsible committee on human experimentation (institutional and national) and with the Helsinki Declaration of 1975, as revised in 2000 (5). Informed consent was obtained from all patients for being included in the study.'

If any identifying information about patients is included in the article, the following sentence should also be included:

'Additional informed consent was obtained from all patients for which identifying information is included in this article.'

Follow the below link for Springer's Informed Consent Statement:

http://www.springer.com/authors?SGWID=0-111-6-608209-0

\section{Animal Studies}

For studies with animals, include the following sentence in the manuscript before the References section:

'All institutional and national guidelines for the care and use of laboratory animals were followed.' 
If the authors did not carry out animal and/or human studies as part of their article they must include the following statement in the manuscript before the References section:

'No animal or human studies were carried out by the authors for this article'

The editors reserve the right to reject manuscripts that do not comply with the abovementioned requirements. The author will be held responsible for false statements or failure to fulfill the above-mentioned requirements

Follow the below link for Springer's Animal and Human Rights Statement:

http://www.springer.com/authors?SGWID=0-111-6-608309-0

\section{ENGLISH LANGUAGE EDITING}

For editors and reviewers to accurately assess the work presented in your manuscript you need to ensure the English language is of sufficient quality to be understood. If you need help with writing in English you should consider:

- Asking a colleague who is a native English speaker to review your manuscript for clarity.

- Visiting the English language tutorial which covers the common mistakes when writing in English.

- Using a professional language editing service where editors will improve the English to ensure that your meaning is clear and identify problems that require your review.

Two such services are provided by our affiliates Nature Research Editing Service and American Journal Experts. Springer authors are entitled to a 10\% discount on their first submission to either of these services, simply follow the links below.

\section{English language tutorial}

\section{Nature Research Editing Service}

\section{$\underline{\text { American Journal Experts }}$}


Please note that the use of a language editing service is not a requirement for publication in this journal and does not imply or guarantee that the article will be selected for peer review or accepted.

If your manuscript is accepted it will be checked by our copyeditors for spelling and formal style before publication.

\section{ETHICAL RESPONSIBILITIES OF AUTHORS}

This journal is committed to upholding the integrity of the scientific record. As a member of the Committee on Publication Ethics (COPE) the journal will follow the COPE guidelines on how to deal with potential acts of misconduct.

Authors should refrain from misrepresenting research results which could damage the trust in the journal, the professionalism of scientific authorship, and ultimately the entire scientific endeavour. Maintaining integrity of the research and its presentation can be achieved by following the rules of good scientific practice, which include:

- The manuscript has not been submitted to more than one journal for simultaneous consideration.

- The manuscript has not been published previously (partly or in full), unless the new work concerns an expansion of previous work (please provide transparency on the reuse of material to avoid the hint of text-recycling ("self-plagiarism")).

- A single study is not split up into several parts to increase the quantity of submissions and submitted to various journals or to one journal over time (e.g. "salami-publishing").

- No data have been fabricated or manipulated (including images) to support your conclusions.

- No data, text, or theories by others are presented as if they were the author's own ("plagiarism"). Proper acknowledgements to other works must be given (this includes material that is closely copied (near verbatim), summarized and/or paraphrased), quotation marks are used for verbatim copying of material, and permissions are 
secured for material that is copyrighted. Important note: the journal may use software to screen for plagiarism.

- Consent to submit has been received explicitly from all co-authors, as well as from the responsible authorities - tacitly or explicitly - at the institute/organization where the work has been carried out, before the work is submitted.

- Authors whose names appear on the submission have contributed sufficiently to the scientific work and therefore share collective responsibility and accountability for the results.

- Authors are strongly advised to ensure the correct author group, corresponding author, and order of authors at submission. Changes of authorship or in the order of authors are not accepted after acceptance of a manuscript. Adding and/or deleting authors at revision stage may be justifiably warranted. A letter must accompany the revised manuscript to explain the role of the added and/or deleted author(s). Further documentation may be required to support your request. Requests for addition or removal of authors as a result of authorship disputes after acceptance are honored after formal notification by the institute or independent body and/or when there is agreement between all authors.

- Upon request authors should be prepared to send relevant documentation or data in order to verify the validity of the results. This could be in the form of raw data, samples, records, etc. Sensitive information in the form of confidential proprietary data is excluded.

If there is a suspicion of misconduct, the journal will carry out an investigation following the COPE guidelines. If, after investigation, the allegation seems to raise valid concerns, the accused author will be contacted and given an opportunity to address the issue. If misconduct has been established beyond reasonable doubt, this may result in the Editor-in-Chief's implementation of the following measures, including, but not limited to:

- If the article is still under consideration, it may be rejected and returned to the author. 
- If the article has already been published online, depending on the nature and severity of the infraction, either an erratum will be placed with the article or in severe cases complete retraction of the article will occur. The reason must be given in the published erratum or retraction note. Please note that retraction means that the paper is maintained on the platform, watermarked "retracted" and explanation for the retraction is provided in a note linked to the watermarked article.

- The author's institution may be informed.

\section{COMPLIANCE WITH ETHICAL STANDARDS}

To ensure objectivity and transparency in research and to ensure that accepted principles of ethical and professional conduct have been followed, authors should include information regarding sources of funding, potential conflicts of interest (financial or non-financial), informed consent if the research involved human participants, and a statement on welfare of animals if the research involved animals.

Authors should include the following statements (if applicable) in a separate section entitled "Compliance with Ethical Standards" when submitting a paper:

- Disclosure of potential conflicts of interest

- Research involving Human Participants and/or Animals

- Informed consent

Please note that standards could vary slightly per journal dependent on their peer review policies (i.e. single or double blind peer review) as well as per journal subject discipline. Before submitting your article check the instructions following this section carefully.

The corresponding author should be prepared to collect documentation of compliance with ethical standards and send if requested during peer review or after publication. 
The Editors reserve the right to reject manuscripts that do not comply with the abovementioned guidelines. The author will be held responsible for false statements or failure to fulfill the abovementioned guidelines.

\section{DISCLOSURE OF POTENTIAL CONFLICTS OF INTEREST}

Authors must disclose all relationships or interests that could have direct or potential influence or impart bias on the work. Although an author may not feel there is any conflict, disclosure of relationships and interests provides a more complete and transparent process, leading to an accurate and objective assessment of the work. Awareness of a real or perceived conflict of interest is a perspective to which the readers are entitled. This is not meant to imply that a financial relationship with an organization that sponsored the research or compensation received for consultancy work is inappropriate. Examples of potential conflicts of interests that are directly or indirectly related to the research may include but are not limited to the following:

- Research grants from funding agencies (please give the research funder and the grant number)

- Honoraria for speaking at symposia

- Financial support for attending symposia

- Financial support for educational programs

- Employment or consultation

- Support from a project sponsor

- Position on advisory board or board of directors or other type of management relationships

- Multiple affiliations

- Financial relationships, for example equity ownership or investment interest

- Intellectual property rights (e.g. patents, copyrights and royalties from such rights)

- Holdings of spouse and/or children that may have financial interest in the work 
In addition, interests that go beyond financial interests and compensation (non-financial interests) that may be important to readers should be disclosed. These may include but are not limited to personal relationships or competing interests directly or indirectly tied to this research, or professional interests or personal beliefs that may influence your research.

The corresponding author collects the conflict of interest disclosure forms from all authors. In author collaborations where formal agreements for representation allow it, it is sufficient for the corresponding author to sign the disclosure form on behalf of all authors. Examples of forms can be found here:

The corresponding author will include a summary statement in the text of the manuscript in a separate section before the reference list, that reflects what is recorded in the potential conflict of interest disclosure form(s).

See below examples of disclosures:

Funding: This study was funded by $\mathrm{X}$ (grant number $\mathrm{X}$ ).

Conflict of Interest: Author A has received research grants from Company A. Author B has received a speaker honorarium from Company $\mathrm{X}$ and owns stock in Company $\mathrm{Y}$. Author $\mathrm{C}$ is a member of committee $Z$.

If no conflict exists, the authors should state:

Conflict of Interest: The authors declare that they have no conflict of interest.

\section{RESEARCH INVOLVING HUMAN PARTICIPANTS AND/OR ANIMALS}

\section{1) Statement of human rights}

When reporting studies that involve human participants, authors should include a statement that the studies have been approved by the appropriate institutional and/or national research ethics committee and have been performed in accordance with the ethical standards as laid down in the 1964 Declaration of Helsinki and its later amendments or comparable ethical standards. 
If doubt exists whether the research was conducted in accordance with the 1964 Helsinki Declaration or comparable standards, the authors must explain the reasons for their approach, and demonstrate that the independent ethics committee or institutional review board explicitly approved the doubtful aspects of the study.

The following statements should be included in the text before the References section:

Ethical approval: "All procedures performed in studies involving human participants were in accordance with the ethical standards of the institutional and/or national research committee and with the 1964 Helsinki declaration and its later amendments or comparable ethical standards."

For retrospective studies, please add the following sentence:

"For this type of study formal consent is not required."

\section{2) Statement on the welfare of animals}

The welfare of animals used for research must be respected. When reporting experiments on animals, authors should indicate whether the international, national, and/or institutional guidelines for the care and use of animals have been followed, and that the studies have been approved by a research ethics committee at the institution or practice at which the studies were conducted (where such a committee exists).

For studies with animals, the following statement should be included in the text before the References section:

Ethical approval: "All applicable international, national, and/or institutional guidelines for the care and use of animals were followed."

If applicable (where such a committee exists): "All procedures performed in studies involving animals were in accordance with the ethical standards of the institution or practice at which the studies were conducted." 
If articles do not contain studies with human participants or animals by any of the authors, please select one of the following statements:

"This article does not contain any studies with human participants performed by any of the authors."

"This article does not contain any studies with animals performed by any of the authors."

"This article does not contain any studies with human participants or animals performed by any of the authors."

\section{INFORMED CONSENT}

All individuals have individual rights that are not to be infringed. Individual participants in studies have, for example, the right to decide what happens to the (identifiable) personal data gathered, to what they have said during a study or an interview, as well as to any photograph that was taken. Hence it is important that all participants gave their informed consent in writing prior to inclusion in the study. Identifying details (names, dates of birth, identity numbers and other information) of the participants that were studied should not be published in written descriptions, photographs, and genetic profiles unless the information is essential for scientific purposes and the participant (or parent or guardian if the participant is incapable) gave written informed consent for publication. Complete anonymity is difficult to achieve in some cases, and informed consent should be obtained if there is any doubt. For example, masking the eye region in photographs of participants is inadequate protection of anonymity. If identifying characteristics are altered to protect anonymity, such as in genetic profiles, authors should provide assurance that alterations do not distort scientific meaning.

The following statement should be included:

Informed consent: "Informed consent was obtained from all individual participants included in the study." 
If identifying information about participants is available in the article, the following statement should be included:

"Additional informed consent was obtained from all individual participants for whom identifying information is included in this article."

\section{RESEARCH DATA POLICY}

A submission to the journal implies that materials described in the manuscript, including all relevant raw data, will be freely available to any researcher wishing to use them for noncommercial purposes, without breaching participant confidentiality.

The journal strongly encourages that all datasets on which the conclusions of the paper rely should be available to readers. We encourage authors to ensure that their datasets are either deposited in publicly available repositories (where available and appropriate) or presented in the main manuscript or additional supporting files whenever possible. Please see Springer Nature's information on recommended repositories.

- $\quad$ List of Repositories

- $\quad$ Research Data Policy

General repositories - for all types of research data - such as figshare and Dryad may be used where appropriate.

Datasets that are assigned digital object identifiers (DOIs) by a data repository may be cited in the reference list. Data citations should include the minimum information recommended by DataCite: authors, title, publisher (repository name), identifier.

- $\quad$ DataCite

Where a widely established research community expectation for data archiving in public repositories exists, submission to a community-endorsed, public repository is mandatory. Persistent identifiers (such as DOls and accession numbers) for relevant datasets must be provided in the paper. 
For more information:

- Research Data Policy Frequently Asked Questions

\section{Data availability}

The journal encourages authors to provide a statement of Data availability in their article. Data availability statements should include information on where data supporting the results reported in the article can be found, including, where applicable, hyperlinks to publicly archived datasets analysed or generated during the study. Data availability statements can also indicate whether data are available on request from the authors and where no data are available, if appropriate.

Data Availability statements can take one of the following forms (or a combination of more than one if required for multiple datasets):

1. The datasets generated during and/or analysed during the current study are available in the [NAME] repository, [PERSISTENT WEB LINK TO DATASETS].

2. The datasets generated during and/or analysed during the current study are not publicly available due [REASON WHY DATA ARE NOT PUBLIC] but are available from the corresponding author on reasonable request.

3. The datasets generated during and/or analysed during the current study are available from the corresponding author on reasonable request.

4. Data sharing not applicable to this article as no datasets were generated or analysed during the current study.

5. All data generated or analysed during this study are included in this published article [and its supplementary information files].

More examples of template data availability statements, which include examples of openly available and restricted access datasets, are available:

- $\quad$ Data availability statements 
Springer Nature provides a research data policy support service for authors and editors, which can be contacted at researchdata@springernature.com.

This service provides advice on research data policy compliance and on finding research data repositories. It is independent of journal, book and conference proceedings editorial offices and does not advise on specific manuscripts.

- $\underline{\text { Helpdesk }}$

\section{AFTER ACCEPTANCE}

Upon acceptance of your article you will receive a link to the special Author Query Application at Springer's web page where you can sign the Copyright Transfer Statement online and indicate whether you wish to order OpenChoice, offprints, or printing of figures in color.

Once the Author Query Application has been completed, your article will be processed and you will receive the proofs.

\section{Copyright transfer}

Authors will be asked to transfer copyright of the article to the Publisher (or grant the Publisher exclusive publication and dissemination rights). This will ensure the widest possible protection and dissemination of information under copyright laws.

- Creative Commons Attribution-NonCommercial 4.0 International License

\section{Offprints}

Offprints can be ordered by the corresponding author.

\section{Color illustrations}

Online publication of color illustrations is free of charge. For color in the print version, authors will be expected to make a contribution towards the extra costs. 


\section{Proof reading}

The purpose of the proof is to check for typesetting or conversion errors and the completeness and accuracy of the text, tables and figures. Substantial changes in content, e.g., new results, corrected values, title and authorship, are not allowed without the approval of the Editor.

After online publication, further changes can only be made in the form of an Erratum, which will be hyperlinked to the article.

\section{Online First}

The article will be published online after receipt of the corrected proofs. This is the official first publication citable with the DOI. After release of the printed version, the paper can also be cited by issue and page numbers.

\section{OPEN CHOICE}

In addition to the normal publication process (whereby an article is submitted to the journal and access to that article is granted to customers who have purchased a subscription), Springer provides an alternative publishing option: Springer Open Choice. A Springer Open Choice article receives all the benefits of a regular subscription-based article, but in addition is made available publicly through Springer's online platform SpringerLink.

\section{- Open Choice}

\section{Copyright and license term - CC BY}

Open Choice articles do not require transfer of copyright as the copyright remains with the author. In opting for open access, the author(s) agree to publish the article under the Creative Commons Attribution License. 


\section{Addendum B: Search Strategy}

\section{Medline (PubMed)}

Date searched: $\quad 29$ March 2017

Results: $\quad 272$ titles

Search terms:

("Upper Extremity/education"[MeSH] OR "Upper Extremity/injuries"[MeSH] OR "Upper Extremity/therapy"[MeSH] OR "Cumulative Trauma Disorders"[MeSH] OR "Arthritis/therapy"[MeSH] OR "Musculoskeletal Diseases/therapy"[MeSH])

AND

("Occupational Therapy"[MeSH] OR "Physical Therapy Modalities/education"[MeSH] OR "Physical Therapy Modalities/rehabilitation"[MeSH] OR "Physical Therapy Modalities/therapy"[MeSH] OR "Human Engineering/therapeutic use"[MeSH] OR "Exercise Therapy"[MeSH] OR "Employment, Supported"[MeSH] OR "Rehabilitation, Vocational"[MeSH] OR stretching[All Fields] OR "rest break*"[All Fields] OR "workstation adjustment*"[All Fields] OR "reasonable accommodation*"[All Fields] OR "modified work"[All Fields] OR "modified job*"[All Fields]))

AND

("Workplace"[MeSH] OR "Industry"[MeSH] OR "Occupational Health"[MeSH] OR "Employment"[MeSH] OR employer[All Fields] OR factory[All Fields] OR factories[All Fields] OR ("Office"[Journal] OR "office"[All Fields]) OR company[All Fields] OR companies[All Fields] OR onsite[All Fields] OR employee[All Fields] OR worker[All Fields] OR ("manpower"[Subheading] OR "manpower"[All Fields] OR "workers"[All Fields])) 
("clinical trial*" OR "randomized controlled trial*" OR "random allocation" OR placebo* OR "random research" OR comparative OR "evaluation stud"” OR "follow up" OR "prospective*" OR "cohort" OR "control*" OR "case series" OR "cross-sectional" OR "experimental stud" OR "single mask" OR "double mask*" OR "treble mask*" OR "triple mask*" OR "single-blind" OR "double-blind*" OR "treble blind*" OR "triple blind*" OR RCT*) 


\section{OTSeeker}

Date searched: $\quad 29$ March 2017

Results: $\quad 172$ titles

Search terms:

("upper extremity" OR "upper limb" OR arm OR axilla OR forearm OR hand or finger OR wrist OR elbow OR shoulder OR "cumulative trauma disorder" OR "repetitive strain injur" OR "compression neuropathy" OR "carpal tunnel" OR "trigger finger" OR "tenosynovitis" OR "tendonitis" OR "tendon injury" OR "tendinopathy" OR "lateral epicondylitis" OR "tennis elbow" OR "rotator cuff" OR "arthritis" OR "soft tissue injur*” OR "cubital tunnel syndrome" OR ganglion cyst OR neuralgia OR neuritis OR bursitis OR arthralgia OR "musculoskeletal" OR "radiculopathy" OR "synovitis")

AND

("vocational rehabilitation" OR rehabilitation" OR "work hardening" OR "intervention*" OR "occupational therapy" OR "physiotherap*" OR "physical therap*" OR "ergonomic*" OR "wellness" OR "exercise" OR "stretching" OR "rest breaks" OR "workstation adjustment" OR "reasonable accommodation*" OR "education" OR "training" OR "supported employment" OR "job rotation" OR "splint"” OR "modified work" OR "modified job*" OR "arm support" OR "wrist guard*")

AND

(worksite OR workplace OR employer OR industry OR factory OR factories OR office OR company OR "occupational health" OR employment OR onsite OR employee OR worker OR workers) 


\section{EBSCOhost}

Date searched: $\quad 29$ March 2017

Databases included: Africa-Wide, Academic Search Premier, CINAHL

Results: $\quad 97$ titles

Limiters applied:

- Boolean phrase

- Peer-reviewed journals only

- Human only, all adults (CINAHL only)

Search terms:

("upper extremity" OR "upper limb" OR arm OR axilla OR forearm OR hand or finger OR wrist OR elbow OR shoulder OR "cumulative trauma disorder" OR "repetitive strain injur" OR "compression neuropathy" OR "carpal tunnel" OR "trigger finger" OR "tenosynovitis" OR "tendonitis" OR "tendon injury" OR "tendinopathy" OR "lateral epicondylitis" OR "tennis elbow" OR "rotator cuff" OR "arthritis" OR "soft tissue injur"” OR "cubital tunnel syndrome" OR ganglion cyst OR neuralgia OR neuritis OR bursitis OR arthralgia OR "musculoskeletal" OR "radiculopathy" OR "synovitis")

AND

("vocational rehabilitation" OR rehabilitation OR "work hardening" OR intervention* OR "occupational therapy" OR physiotherap OR "physical therap*" OR ergonomic* OR "wellness" OR "exercise" OR "stretching" OR "rest breaks" OR "workstation adjustment*" OR "reasonable accommodation*" OR "education" OR "training" OR "supported employment" OR "job rotation" OR "splint" OR "modified work" OR “modified job"” OR "arm support" OR "wrist guard")

AND 
(worksite OR workplace OR employer OR industry OR factory OR factories OR office OR company OR "occupational health" OR employment OR onsite OR employee OR worker OR workers)

AND

("clinical trial*" OR "randomized controlled trial*" OR "random allocation" OR placebo* OR "random research" OR comparative OR "evaluation stud*” OR "follow up" OR "prospective*" OR "cohort" OR "control*" OR "case series" OR "cross-sectional" OR "experimental stud"” OR "single mask"" OR "double mask*" OR "treble mask" OR "triple mask" OR "single-blind*" OR "double-blind*" OR "treble blind*" OR "triple blind*" OR RCT*) 


\section{Cochrane Library}

Date searched: $\quad 31$ March 2017

Results: $\quad 50$ titles

Search terms:

1. MeSH descriptor: [Upper Extremity] explode all trees

2. MeSH descriptor [Cumulative Trauma Disorders] explode all trees

3. MeSH descriptor [Hand Deformities] explode all trees

4. MeSH descriptor: [Joint Diseases] explode all trees

5. MeSH descriptor [Muscular Diseases] 1 tree(s) exploded

6. MeSH descriptor [Tennis Elbow] explode all trees

7. MeSH descriptor: [Rheumatic Diseases] this term only

AND

8. MeSH descriptor [Occupational Therapy] explode all trees

9. MeSH descriptor [Physical Therapy Modalities] explode all trees

10. MeSH descriptor: [Human Engineering] explode all trees

11. MeSH descriptor: [Employment, Supported] explode all trees

AND

12. MeSH descriptor [Workplace] this term only

13. MeSH descriptor [Industry] explode all trees

14. MeSH descriptor: [Occupational Health] explode all trees

15. MeSH descriptor: [Workers' Compensation] this term only 


\section{Web of Science}

Date searched: $\quad 24$ April 2017

Results: $\quad 50$ titles

Limiters applied: Articles only

Search terms:

( $\mathrm{TI}=$ (“upper extremity" OR "upper limb" OR arm OR axilla OR forearm OR hand or finger OR wrist OR elbow OR shoulder OR "cumulative trauma disorder" OR "repetitive strain injur" OR "compression neuropathy" OR "carpal tunnel" OR "trigger finger" OR "tenosynovitis" OR "tendonitis" OR "tendon injury*" OR "tendinopathy" OR "lateral epicondylitis" OR "tennis elbow" OR "rotator cuff" OR "arthritis" OR "soft tissue injur*" OR "cubital tunnel syndrome" OR ganglion cyst OR neuralgia OR neuritis OR bursitis OR arthralgia OR "musculoskeletal" OR "radiculopathy" OR "synovitis")

\section{AND}

$\mathrm{TI}=$ (“vocational rehabilitation" OR rehabilitation OR "work hardening" OR intervention* OR "occupational therapy" OR physiotherap OR "physical therap*" OR ergonomic* OR wellness OR exercise OR stretching OR "rest breaks" OR "workstation adjustment*" OR "reasonable accommodation*" OR education OR training OR "supported employment" OR "job rotation" OR splint* OR “modified work" OR "modified job*” OR "arm support" OR "wrist guard*”)

\section{AND}

$\mathrm{TI}=$ (worksite OR workplace OR employer OR industry OR factory OR factories OR office OR company OR "occupational health" OR employment OR onsite OR employee OR worker OR workers)

AND 
$\mathrm{TI}=($ "clinical trial"* OR "randomized controlled trial*" OR "random allocation" OR placebo* OR "random research" OR comparative OR "evaluation stud"” OR "follow up" OR "prospective*" OR "cohort" OR "control*" OR "case series" OR "cross-sectional" OR "experimental stud" OR "single mask" OR "double mask*" OR "treble mask" OR "triple mask *" OR "single-blind" OR "double-blind"" OR "treble blind" OR "triple blind*" OR $\left.\mathrm{RCT}^{*}\right)$ ) AND DOCUMENT TYPES: (Article) 


\section{Scopus}

Date searched: $\quad 25$ April 2017

Results: $\quad 420$ titles

Limiters applied: $\quad$ Articles only

Search terms:

TITLE-ABS ( "upper extremity" OR "upper limb" OR arm OR axilla OR forearm OR hand OR finger OR wrist OR elbow OR shoulder OR "cumulative trauma disorder" OR repetitive strain injur" OR "compression neuropathy" OR "carpal tunnel" OR "trigger finger" OR tenosynovitis OR tendonitis OR "tendon injury" OR tendinopathy OR "lateral epicondylitis" OR "tennis elbow" OR "rotator cuff" OR "soft tissue injur" OR "cubital tunnel syndrome" OR ganglion OR cyst OR neuralgia OR neuritis OR bursitis OR musculoskeletal OR radiculopathy OR synovitis )

AND

TITLE-ABS ("vocational rehabilitation" OR rehabilitation OR "work hardening" OR intervention* OR "occupational therapy" OR physiotherapy* OR "physical therap" OR ergonomic* OR wellness OR exercise OR stretching OR "rest breaks" or "workstation adjustment" OR "reasonable accommodation" Or education OR training OR "supported employment" OR "job rotation" OR splint* OR "modified work" OR "modified job" Or "arm support" OR "wrist guard*”)

$A N D$

TITLE-ABS ( worksite OR workplace OR employer OR industry OR factory OR factories OR office OR company or "occupational health" OR employment OR onsite OR employee OR worker OR workers )

AND 
TITLE-ABS ("clinical trial*" OR "randomized controlled trial" OR "random allocation" OR placebo" OR "random research" OR comparative OR "evaluation stud" Or "follow up" OR prospective* OR cohort OR control* Or "case series" Or cross-sectional" OR "experimental stud" Or "single mask" OR "double mask" Or "treble mask" OR "triple mask" OR "singleblind" OR "double-blind"” OR "treble blind"” OR "triple blind"” OR rct" )

AND ( LIMIT-TO (SRCTYPE, "j")) AND (LIMIT-TO (DOCTYPE, "ar ")) 


\section{PEDro}

Date searched: $\quad 28$ April 2017

Results:

11 titles

Type of search: Simple search

Search terms:

Workplace (upper limb) 


\section{Addendum C: Quality appraisal table}

\begin{tabular}{|c|c|c|c|c|c|c|c|c|c|c|c|c|c|c|c|c|c|c|c|}
\hline & 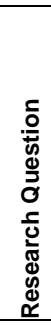 & 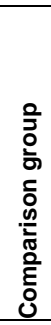 & 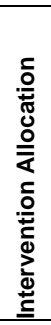 & 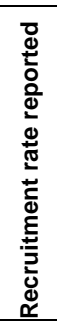 & 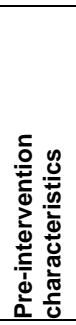 & 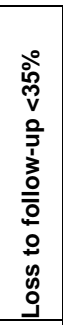 & 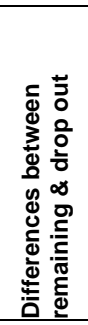 & 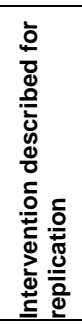 & 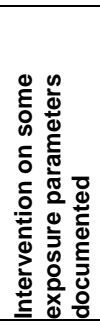 & 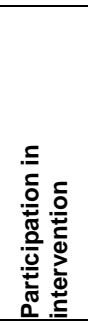 & 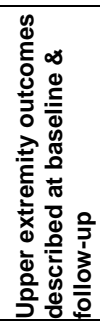 & 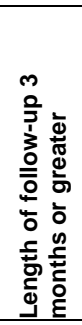 & 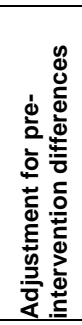 & 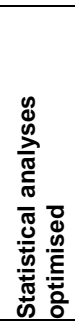 & 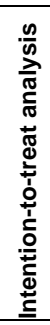 & 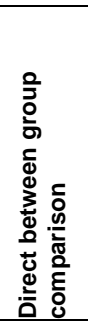 & 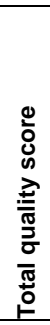 & 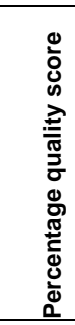 & 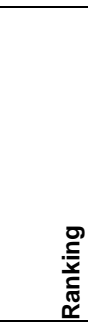 \\
\hline Criteria Code & 1 & 2 & 3 & 4 & 5 & 6 & 7 & 8 & 9 & 10 & 11 & 12 & 13 & 14 & 15 & 16 & & & \\
\hline Weight & 2 & 3 & 3 & 2 & 2 & 2 & 2 & 3 & 1 & 2 & 3 & 2 & 3 & 3 & 2 & 3 & & & \\
\hline Max Score & 1 & 1 & 2 & 1 & 1 & 1 & 1 & 1 & 1 & 1 & 1 & 1 & 1 & 1 & 1 & 1 & 41 & & \\
\hline $\begin{array}{l}\text { Main article } \\
\text { Author: year }\end{array}$ & & & & & & & & & & & & & & & & & & & \\
\hline Aaras: 1999 & 1 & 1 & 2 & 1 & 1 & 1 & 1 & 1 & 1 & 0 & 1 & 1 & 1 & 0 & 1 & 0 & 33 & $80 \%$ & Medium \\
\hline Andersen, L: 2011 & 1 & 1 & 2 & 1 & 1 & 1 & 0 & 1 & 1 & 1 & 1 & 0 & 1 & 1 & 1 & 1 & 37 & $90 \%$ & High \\
\hline Andersen, C: 2011 & 1 & 1 & 2 & 1 & 1 & 1 & 0 & 1 & 0 & 1 & 1 & 0 & 1 & 1 & 1 & 1 & 36 & $88 \%$ & High \\
\hline Bernaards: 2006 & 1 & 1 & 2 & 1 & 1 & 1 & 1 & 1 & 1 & 1 & 1 & 1 & 1 & 1 & 1 & 1 & 41 & $100 \%$ & High \\
\hline Camargo: 2009 & 1 & 0 & 1 & 1 & 0 & 1 & 0 & 1 & 0 & 0 & 1 & 0 & 0 & 0 & 0 & 0 & 15 & $37 \%$ & Low \\
\hline Cheng: 2007 & 1 & 1 & 2 & 1 & 1 & 1 & 0 & 1 & 1 & 0 & 1 & 0 & 1 & 1 & 0 & 1 & 33 & $80 \%$ & Medium \\
\hline Dropkin: 2015 & 1 & 1 & 2 & 1 & 1 & 1 & 1 & 1 & 1 & 0 & 1 & 1 & 1 & 1 & 1 & 1 & 39 & $95 \%$ & High \\
\hline Esmaeilzadeh: 2014 & 1 & 1 & 2 & 1 & 1 & 1 & 0 & 0 & 1 & 0 & 1 & 1 & 1 & 1 & 0 & 1 & 32 & $78 \%$ & Medium \\
\hline Feuerstein: 2004 & 1 & 1 & 2 & 1 & 1 & 1 & 1 & 1 & 1 & 0 & 1 & 1 & 0 & 1 & 1 & 1 & 36 & $88 \%$ & High \\
\hline Hagberg: 2000 & 0 & 1 & 2 & 0 & 1 & 1 & 0 & 1 & 0 & 1 & 1 & 1 & 0 & 1 & 0 & 1 & 29 & $71 \%$ & Medium \\
\hline Jay: 2014 & 0 & 1 & 2 & 1 & 1 & 1 & 1 & 1 & 1 & 1 & 1 & 0 & 1 & 1 & 1 & 1 & 37 & $90 \%$ & High \\
\hline Lincoln: 2002 & 1 & 1 & 2 & 1 & 1 & 0 & 0 & 1 & 0 & 0 & 0 & 0 & 0 & 0 & 0 & 1 & 21 & $51 \%$ & Medium \\
\hline Lundblad: 1999 & 1 & 1 & 2 & 1 & 1 & 0 & 1 & 1 & 1 & 1 & 1 & 1 & 0 & 1 & 1 & 1 & 36 & $88 \%$ & High \\
\hline Martimo: 2010 & 1 & 1 & 2 & 1 & 1 & 0 & 1 & 0 & 0 & 0 & 1 & 0 & 1 & 1 & 1 & 1 & 31 & $76 \%$ & Medium \\
\hline Ripat: 2006 & 1 & 1 & 2 & 0 & 1 & 1 & 1 & 1 & 1 & 1 & 1 & 1 & 1 & 0 & 0 & 0 & 31 & $76 \%$ & Medium \\
\hline Sundstrup: 2013 & 1 & 1 & 2 & 1 & 1 & 1 & 0 & 1 & 1 & 1 & 1 & 0 & 1 & 1 & 1 & 1 & 37 & $90 \%$ & High \\
\hline Voerman: 2007 & 1 & 1 & 2 & 0 & 1 & 1 & 1 & 1 & 0 & 0 & 1 & 1 & 1 & 1 & 1 & 1 & 36 & $88 \%$ & High \\
\hline
\end{tabular}

